\title{
Kapitel 4: Die syntaktische Theorie der Beobachtungen
}

\section{Einleitung}

In diesem Kapitel soll es um eine Position zur Theoriebeladenheit der Beobachtung gehen, die in zentralen Punkten auf Paul Feyerabend zurückgeht und von Paul Churchland weiter ausgearbeitet wurde. Wie im vorigen Kapitel werde ich mich in der Rekonstruktion aber wieder daran orientieren, eine aus systematischer Sicht möglichst interessante Position zu formulieren. Weitere Überlegungen der Autoren zur Theoriebeladenheit werden, soweit sie nicht schon im vorigen Kapitel angesprochen wurden, im nächsten Kapitel behandelt werden.

Die Position wird grob durch die folgenden beiden Thesen charakterisiert, die im Vergleich zur Position des vorigen Kapitel sowohl allgemeiner als auch inhaltlich stärker sind:

1. Beobachtungen sind im Allgemeinen als Prozesse auffassbar, die zu Sätzen als wissenschaftlich relevantem Produkt führen.

2. Die Bedeutung der Begriffe in Beobachtungssätzen wird durch das Netz theoretischer Überzeugungen bestimmt, in dem diese Begriffe stehen.

Die Thesen sind zunächst allgemeiner als die des vorigen Kapitels, weil sie nicht auf Wahrnehmungen beschränkt sind, sondern alle Formen der Beobachtung betreffen. So sollen sie insbesondere auch für Beobachtungen mit Hilfe von Messgeräten gelten. Die Allgemeinheit der Thesen wird möglich, weil Feyerabend und Churchland ein generelles Modell für Beobachtungen zugrunde legen, das eine einheitliche Beschreibung aller Beobachtungen sowie eine generelle Argumentation für ihre Theorieabhängigkeit erlauben soll. Diesem Modell zufolge sind Beobachtungssätze das in allen Fällen wissenschaftlich relevante Ergebnis eines Beobachtungsprozesses. Dieses Modell für Beobachtungen, das in der ersten These angedeutet ist, werde ich in Abschnitt 2 ausführen und diskutieren.

Die Theorieabhängigkeit von Beobachtungen soll sich dann gemäß der zweiten These vor allem durch die theoretische Bestimmung der Bedeutung von Beobachtungssätzen ergeben. Die hier von den Autoren vertretene semantische Theorie, eine Begriffliche-Rollen-Semantik bzw. NetzwerkSemantik, und die dafür vorgebrachten Gründe werde ich in Abschnitt 3 diskutieren.

Feyerabend bezeichnet das Bild von Beobachtungen, das sich so insgesamt ergibt, als „pragmatische Theorie der Beobachtung“.. Wie aus den Ausfüh-

1 Siehe Feyerabend (1962), 36. 
rungen in diesem Kapitel deutlich werden wird, ist die Bezeichnung ,syntaktische Theorie“ aber angemessener. Denn im Kern wird der Beitrag von Beobachtungen zur wissenschaftlichen Erkenntnisgewinnung auf die Festlegung der Syntax von Beobachtungssätzen beschränkt. Im letzten Abschnitt wird zu fragen sein, ob und auf welche Weise diese Position einen Pessimismus stützt.

Diese Position zur Theorieabhängigkeit von Beobachtungen ist stärker als die des vorigen Kapitels. Der Position zufolge wird der gesamte Inhalt dessen, was an Beobachtungen wissenschaftlich bedeutsam ist, durch Theorien festgelegt. Die Position des vorigen Kapitels sah dagegen vor, dass der Inhalt von Beobachtungen partiell empirisch und partiell theoretisch festgelegt ist. Die dort angeführten Gründe stützen nur diese schwächere Fassung. Inbesondere ist eine Wahrnehmung gemäß einer Helmholtz'schen Wahrnehmungstheorie zum einen zwar durch bestehende (theoretische) Annahmen, zum anderen aber durch die Sinnesreize kausal bestimmt.

Für die Position dieses Kapitels wird dagegen nicht die kausale Genese von Beobachtungsergebnissen angeführt, sondern die Konstitution ihres Inhalts. Es wird zum einen angenommen, dass Theorien den Inhalt von Beobachtungssätzen vollständig konstituieren. Zum anderen sollen neben Beobachtungssätzen keine anderen Stufen oder Produkte von Beobachtungsprozessen wissenschaftlich bedeutsam sein.

Zwar wird eine Netzwerk-Semantik nicht nur von Feyerabend und Churchland, sondern auch von Hanson und Kuhn für die Theoriebeladenheit von Beobachtungen angeführt. ${ }^{2}$ Aber nur die erstgenannten Autoren nehmen eine vollständige theoretische Determination der Beobachtungsinhalte an. Daher ist verständlich, wenn Feyerabend seine Position von solchen anderen abgrenzt, indem er schreibt: ${ }^{3}$

[O]bservations (observation terms) are not merely theory-laden (the position of Hanson, Hesse and others) but fully theoretical (observation statements have no ,observational core). (Feyerabend 1981, x; Feyerabends Hervorhebungen.)

Um die Diskussion überschaubar zu halten, werde ich in diesem Kapitel eine besondere Dimension von Beobachtungen außer Acht lassen. Beobachtungsergebnisse können Hypothesen nur prüfen und haben so Auswirkungen auf die Annahme wissenschaftlicher Theorien, wenn sie wissenschaftlich auch als gültig akzeptiert sind und insbesondere für verlässlich gehalten werden. Eine solche Einschätzung der Verlässlichkeit stellt eine zusätzliche Hürde für die epistemische Wirksamkeit von Beobachtungen dar. Die Theoriebeladen-

2 Für Hanson siehe Kap. 3, Abschn. 4b(i); siehe Kuhn (1970), $258 f f$.

3 Vgl. Churchland (1979), 21/22. 
heit, die sich aus dieser Einschätzung ergeben kann, werde ich erst im nächsten Kapitel diskutieren.

\section{Ein allgemeines Modell für Beobachtungen}

Feyerabend und Churchland vertreten eine einfache Ansicht darüber, was Beobachtungen im Allgemeinen sind. ${ }^{4}$ Demnach sind Beobachtungen Kausalprozesse, die von Umständen der Welt über variable Zwischenzustände zu Sätzen (bzw. genauer zu Satztoken) führen. Die Sätze werden zudem als einzige Resultate der Beobachtungsprozesse in Betracht gezogen, die für eine weitere wissenschaftliche Verwendung relevant sein können. Sätze sind hierbei in sehr weitem Sinn zu verstehen; wie wir sehen werden, betrachten Feyerabend und Churchland bei verschiedenen Beobachtungsarten recht unterschiedliche Dinge als Sätze. Zentral ist jeweils, dass die Beobachtungsergebnisse eine syntaktische Struktur haben und dass ihr Inhalt begrifflich und insgesamt wahrheitswertfähig, also propositional, ist.

Interessant ist hier zunächst, wie diese allgemeine Konzeption von Beobachtungen zu den verschiedenen Arten von Beobachtungen passt, die in den Wissenschaften vorkommen. Feyerabend und Churchland nehmen vor allem zwei Typen von Prozessen in den Blick, die (in einem weiten Sinn) als Beobachtungen gelten können, erstens die Feststellung irgendwelcher Sachverhalte mit Hilfe von Messgeräten und zweitens die sinnliche Wahrnehmung von etwas. Wichtig ist dabei, dass Beobachtungen mit Messgeräten als Modell auch für Beobachtungen durch Wahrnehmungen aufgefasst werden. Zentrale Aspekte von Messungen finden sich Feyerabend und Churchland zufolge auch bei Wahrnehmungen.

Eine Beobachtung mit Hilfe eines Messgeräts (etwa eines Strommessgeräts oder eines Quecksilberthermometers) lässt sich oft in mindestens zwei Schritte einteilen. Erstens führt ein kausaler Prozess vom (vermeintlichen) Untersuchungsgegenstand über innere Zustände des Messgeräts zu einem sichtbaren Zustand dieses Geräts, etwa einem Zeigerstand oder einer bestimmten Höhe der Quecksilbersäule. Zweitens muss dieser Zustand interpretiert werden, etwa als „Es fließt ein Strom von $5 \mathrm{~mA}$ “ oder „Temperatur $20^{\circ} \mathrm{C}^{\prime \prime}$. In diesem zweiten Schritt wird also dem sichtbaren Zustand des Messgeräts ein Satz (bzw. eine Proposition) zugewiesen. Dieser Satz ist dann als wissenschaftlicher Befund verwertbar.

Zwar fallen in der Praxis beide Schritte oft zusammen. In der Regel benutzt man Geräte, deren Skala schon beschriftet ist. Viele Geräte arbeiten auch

4 Für das Folgende siehe Feyerabend (1958), Abschn. 2; Feyerabend (1962), 36-39; Feyerabend (1975), 92-96; Churchland (1979), Kap. 2, insbes. \5. 
digital und zeigen als sichtbare Zustände sowieso nur Sätze (oder Teilsätze) an, ein digitales Amperemeter beispielsweise ,5mA“. Die Trennung der beiden Schritte ist aber analytisch sinnvoll. Denn man muss Messgeräte zuerst kalibrieren, indem man eine Skala anbringt oder einstellt, und man kann sie auch rekalibrieren, indem man beispielsweise die Beschriftung der Skala ändert. Messgeräte, die nicht kalibriert sind, führen zwar oft zu sichtbaren Zuständen, diese sind aber als solche wissenschaftlich nicht verwendbar. Erst die Abbildung der Zustände auf Sätze macht den ablaufenden Prozess zu einem, dessen Ergebnis wissenschaftlich als Beobachtung verwertbar ist. Im Fall der Rekalibrierung bleiben die Abläufe im ersten Teil des Prozesses unverändert, aber die Zuordnung zu Sätzen wird neu vorgenommen. In gleicher Weise ändert sich der potenzielle Beitrag der Beobachtung zur Forschung. ${ }^{5}$

Beim zweiten Schritt selbst kann man wiederum zwei Aspekte unterscheiden. Zum einen wird einem Zeigerstand ein Satz, bloß syntaktisch spezifiziert, zugewiesen. Zum anderen hat dieser Satz eine bestimmte Bedeutung. Wenn man eine Skala mit den Ausdrücken ,5 ... 10 ... $15 \mathrm{~mA}^{\prime}$ beschriftet, haben die Ausdrücke zwar bereits eine Bedeutung. Diese kann sich aber ändern, etwa indem man zu einem neuen Verständnis von Stromstärke kommt. Dann ändert sich die Bedeutung der Sätze, die produziert werden; davon bleibt aber der Prozess, der im Messgerät bis zu den bloß syntaktisch spezifizierten Sätzen abläuft, unberührt. Man könnte diese Auffassung so veranschaulichen:

Messung: ‥ $\quad$ Zeigerstand

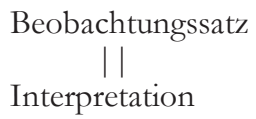

Ein wissenschaftlich relevantes Ergebnis erhält man erst, indem man dem Zeigerstand einen Beobachtungssatz zuordnet, der eine Interpretation hat. Dabei macht sich die Zuordnung zunächst nur an der Syntax des Satzes fest. Auch wenn sich das Verständnis der Ausdrücke ändert, bleibt die Relation zwischen Zeigerstand und Beobachtungssatz bestehen, solange man nicht die Beschriftung des Geräts ändert.

Man könnte einwenden, dass in dieser Beschreibung von Beobachtungen mit Messgeräten mindestens drei Aspekte fehlen. Erstens möchte man nicht beliebige zugeordnete Sätze wirklich wissenschaftlich als Beobachtungen gebrauchen. Vielmehr ist man an solchen Sätzen interessiert, die verlässlich die Umstände angeben, die man mit Hilfe der Messung untersuchen möchte. Man muss daher einschätzen, durch welche Kalibrierung das Gerät zuverläs-

5 Vgl. Feyerabend (1962), 36/37 u. Churchland (1979), 38. 
sig funktioniert. Feyerabend und Churchland ziehen diesen Aspekt in Betracht, ich werde ihn aber hier weitgehend heraushalten. Denn eine solche Einschätzung der Zuverlässigkeit ist in der Regel selbst wieder von theoretischem Wissen abhängig. Wie schon angekündigt, möchte ich diese Form der Theorieabhängigkeit im nächsten Kapitel separat betrachten.

Zweitens könnte man einwenden, dass auch uninterpretierte Messgerätszustände häufig schon gehaltvoll sind. Hierbei könnte man auf Dretskes Explikation der Information, die ein Signal trägt, zurückgreifen. Demnach trägt ein Messgerätszustand grob gesprochen die Information, dass ein bestimmter Sachverhalt besteht, sofern es nomologisch notwendig ist, dass der Sachverhalt besteht, wenn das Gerät in diesem Zustand ist. ${ }^{6}$ Demnach steht ein bestimmter Zeigerstand beispielsweise für die Stromstärke von $5 \mathrm{~mA}$, wenn er nur durch einen solchen Strom verursacht werden kann. Dieser Inhalt ist unabhängig davon, ob die Skala auch entsprechend beschriftet ist. Aber auch wenn man diese Zuschreibung von Inhalten akzeptiert, ${ }^{7}$ ist offensichtlich, dass diese Inhalte von Zeigerständen als solche wissenschaftlich nichts austragen. Zeigerstände sind als solche ,stumm', wie Churchland es ausdrückt. ${ }^{8}$ Man muss erst versuchen, die Ursache des Zeigerstands explizit zu machen, um zu einem wissenschaftlich verwendbaren Ergebnis zu gelangen. Das bedeutet aber gerade, dass man den Zeigerstand interpretiert.

Drittens muss jemand die Sätze auf dem kalibrierten und skalierten Messgerät auch ablesen, damit sie wirklich wissenschaftlich wirksam werden können. Dieser Schritt wird von Feyerabend und Churchland meist übergangen, was aber meiner Einschätzung nach nicht dazu führt, das entworfene Bild nutzlos zu machen. Das Ablesen selbst ist meist unproblematisch. Das kallibrierte Messgerät zeigt mehr oder weniger deutlich einen Satz an. Bei digitalen Geräten muss man ihn einfach lesen. In anderen Fällen muss man ihn aus Zeigerstand und Skala rekonstruieren. In beiden Fällen liegt aber das eigentliche Beobachtungsergebnis schon begrifflich gefasst vor. Daher macht es guten Sinn, nur den Messprozess als wesentlichen Bestandteil des Beobachtungsprozesses aufzufassen und das Ablesen hier außer Betracht zu lassen. (Zur Abgrenzung von Ablesen und Beobachten siehe auch Kapitel 6, Abschn. 2a.)

Der solcherart analysierte Messprozess dient Feyerabend und Churchland als Modell, um auch Wahrnehmungen deskriptiv zu erfassen. Ihnen zufolge sind bei Wahrnehmungen - zumindest insoweit sie wissenschaftliche Beob-

6 Siehe Dretske (1981); vgl. Beckermann (2001), 334/335.

7 Churchland erkennt grundsätzlich solche Inhalte an. Siehe die Unterscheidung von objektiver und subjektiver Intentionalität, Churchland (1979), 14.

8 Siehe Churchland (1979), 38. 
achtungen sein können - ebenfalls zwei Teilprozesse unterscheidbar. Der erste Teilprozess führt von (vermeintlichen) Umständen der Welt über Reizungen der Sinnesorgane zu Empfindungen (,sensations"). Diesen Empfindungen werden dann im zweiten Teilschritt ,Beobachtungssätze zugeordnet. ${ }^{9}$ Zunächst ist es hilfreich, sich etwas mehr Klarheit darüber zu verschaffen, was die Autoren unter Empfindungen und Beobachtungssätzen verstehen. Dann kann man verdeutlichen, welches allgemeine Bild von Beobachtungen sich aus der Analogie von Wahrnehmungen und Messungen ergibt.

Feyerabend und Churchland lassen keine Zweifel daran, dass sie Empfindungen als rein sinnliche Zustände auffassen, die zunächst nur durch ihre phänomenale Qualität charakterisiert sind, d.h. durch die Weise, wie es sich anfühlt, sie zu haben. ${ }^{10}$ Dies bedeutet, dass erstens Empfindungen bewusste Aspekte des Wahrnehmungsprozesses sein sollen, zweitens Empfindungsarten durch rein intrinsische Eigenschaften individuiert werden. Dieses Verständnis von Empfindungen lässt es offen, welche Inhalte eine Wahrnehmung hat, die von bestimmten Empfindungen begleitet wird. Wie wir sehen werden, sind Feyerabend und Churchland der Ansicht, dass Empfindungen weder selbst Inhalte besitzen noch durch ihren phänomenalen Charakter etwas zum Inhalt der Wahrnehmungen beitragen.

Als zweites Element des Wahrnehmungsprozesses machen Feyerabend und Churchland Beobachtungssätze aus. Zwar vertritt Churchland auch die Ansicht, dass die Kategorie ,Beobachtungssatz' letztlich inadäquat ist und durch eine andere Kategorie ersetzt werden muss. Wie sich aber zeigen wird, verändert diese zusätzliche Annahme die gegenwärtig diskutierte Position nicht grundlegend. Vorkommnisse von Beobachtungssätzen sind in Feyerabends und Churchlands Bild von Wahrnehmungen zunächst kausale Produkte des Wahrnehmungsprozesses und folgen in diesem auf Empfindungen. Dabei sind sie aber in der Regel keine öffentlichen sprachlichen Äußerungen,

9 Siehe Feyerabend (1958), 7; Feyerabend (1961), 83; Churchland (1979), Kap. 2. Feyerabend (1962) spricht statt von Empfindungen auch von einem sich charakteristisch anfühlenden Drang, einen Satz zu äußern: „... the urge we feel under certain circumstances to say ,I am in pain' and the peculiar character of this urge (it is different from the urge we feel when we say ,I am hungry")..." (Feyerabend 1962, 38).

Insbesondere Churchland spricht auch oft von Beobachtungsurteilen statt von Sätzen. Ich werde aber durchgehend von Sätzen sprechen, da das Verhältnis dieser Sätze gemäß Churchlands und Feyerabends Verständnis zu den Beobachtungsurteilen, wie sie in Kap. 3, Abschn. 2 besprochen wurden, problematisch ist. Hierzu gleich mehr.

10 Siehe etwa Churchland (1979), 15, und Feyerabend (1962), die in der vorigen Fußnote zitierte Stelle. 
sondern mentale Zustände. Trotzdem werden sie im Übrigen weitgehend als Sätze im sprachlichen Sinn aufgefasst, d.h. man kann fragen, wie sie syntaktisch strukturiert sind, welche Bedeutung sie haben und wodurch diese festgelegt wird, und unter welchen Umständen wir dazu neigen, die Sätze zu produzieren. Wenn sie bedeutungsvoll sind, haben sie propositionalen Gehalt, d.h. ihr Inhalt ist begrifflich.

Es lohnt sich, genauer zu fragen, was hier mit Beobachtungssatz gemeint ist. Zum einen kann man fragen, welche Aspekte der komplexen Phänomenologie von Wahrnehmungen damit beschrieben werden sollen. Zum anderen ist $\mathrm{zu}$ untersuchen, was mit dieser Beschreibung über Wahrnehmungen behauptet wird und wie adäquat dies ist. Ein Beispiel kann helfen festzustellen, welcher Aspekt der Wahrnehmung von Feyerabend und Churchland als Beobachtungssatz bezeichnet wird.

Angenommen, es sieht für mich so aus, als wäre da ein Lindenbaum. Das heißt, ich habe bestimmte Empfindungen, überwiegend Lindgrün-Empfindungen. Ich sehe das Objekt meiner Wahrnehmung zudem als Lindenbaum, d.h. ich klassifiziere es perzeptuell entsprechend. Nehmen wir aber zusätzlich an, dass ich nicht perzeptuell urteile, dass da ein Lindenbaum ist. Denn ich weiß, dass ich mich in einem Freizeitpark befinde, in dem ausschließlich Plastikattrappen von Bäumen aufgestellt sind. Ich komme daher visuell zur Überzeugung, dass da eine Lindenbaum-Attrappe steht. Was ist in dieser Wahrnehmung der Beobachtungssatz?

Es gibt hier zwei Optionen. Der ersten Option zufolge ist erst das eigentliche Beobachtungsurteil ein Beobachtungssatz, die zugrunde liegende und inhaltlich abweichende perzeptuelle Klassifikation ist kein solcher Satz. Dann ist aber Feyerabends und Churchlands Beschreibung von Wahrnehmungsprozessen durch Empfindung und Beobachtungssatz nicht erschöpfend. Sie lässt eine Stufe außer Acht, die gehaltvoll und daher erkenntnistheoretisch von außerordentlicher Bedeutung ist. Eine solchermaßen lückenhafte Beschreibung stellt aber keine brauchbare Grundlage für eine erkenntnistheoretische Diskussion von Beobachtungen dar.

Man muss daher annehmen, dass Feyerabend und Churchland der zweiten Option folgen. Gemäß dieser Option stellt schon die perzeptuelle Klassifikation als Lindenbaum, also der Umstand, dass mir etwas wie ein Lindenbaum visuell erscheint, die Produktion eines Beobachtungssatzes dar. ${ }^{11}$ Möglicherweise wird man das abweichende Beobachtungsurteil als gesonderten Beobachtungssatz betrachten müssen, eher wird man es jedoch als Schluss aus dem zugrunde liegenden Beobachtungssatz und dem Wissen um die besondere

11 Vgl. hierzu Feyerabend (1975), 92/93. 
Umgebung rekonstruieren. Wichtig ist aber, dass Feyerabend und Churchland davon ausgehen müssen, dass die Kategorie ,Beobachtungssatz' den Inhalt perzeptueller Erfahrungen vollständig erfassen muss. Denn Inhalte von Wahrnehmungen sind ganz offensichtlich erkenntnistheoretisch bedeutsam, und die Autoren haben nur eine einzige Kategorie, um diese Inhalte zu beschreiben, die des Beobachtungssatzes. Dies muss im Folgenden im Auge behalten werden. Wenn es etwa um die Frage geht, wodurch der Inhalt von Beobachtungssätzen festgelegt wird, kann eine Position, die für Sätze im üblichen Sinn plausibel erscheint, sich als fragwürdig oder falsch herausstellen, wenn man sich klarmacht, was mit der Kategorie „Beobachtungssatz“ eigentlich beschrieben wird.

Feyerabend und Churchland zufolge macht sich die Kausalrelation zwischen Empfindungen und Satz zunächst an der Syntax des Satzes fest, sie besteht daher schon allein kraft der syntaktischen Eigenschaften der Sätze. Dies kann man zugeben, ohne der Debatte um die Semantik für Beobachtungssätze vorzugreifen, die im nächsten Abschnitt aufgenommen werden soll. Denn man kann hier die beiden Fragen unterscheiden, erstens woran sich die kausalen Relationen zwischen mentalen Zuständen festmachen, und zweitens, wodurch der Inhalt der mentalen Zustände festgelegt wird. Es ist nicht unplausibel (und alles andere als ungewöhnlich) anzunehmen, dass die kausalen Relationen zwischen mentalen Zuständen sich an deren syntaktischer Struktur festmachen. Diese Ansicht ist aber mit sehr unterschiedlichen semantischen Positionen vereinbar. Man kann einerseits der Ansicht sein, dass die kausalen Relationen die Bedeutung der Zustände mit festlegen. Andererseits kann man aber auch wie Feyerabend und Churchland behaupten, dass durch das Bestehen kausaler Relationen noch nichts über den Inhalt der Sätze festgelegt wird. Dann ist bei Wahrnehmungen wie bei Messgeräten zu erwarten, dass die Inhalte der Beobachtungssätze sich radikal ändern können, während deren Verursachungsrelationen bestehen bleiben.

Wie schon angedeutet, vertritt Churchland auch die Ansicht, dass die gehaltvollen mentalen Wahrnehmungszustände letztlich nicht adäquat als Sätze beschrieben werden können, auch wenn er selbst diese Beschreibung häufig verwendet. Stattdessen sollten sie Churchland zufolge als mentale Repräsentationen im Sinne des Konnektionismus aufgefasst werden, die keine satzartige Struktur haben. ${ }^{12}$ Doch auch vor dem Hintergrund dieser Position ergibt sich, dass die mentalen Repräsentationen schon allein kraft ihrer strukturellen, nicht aber ihrer semantischen Eigenschaften in Kausalrelationen

12 Siehe etwa Churchland (1990). Mehr zum Churchland'schen Konnektionismus im nächsten Abschnitt. 
zu anderen mentalen Zuständen stehen. Zudem sollen die Inhalte der Repräsentationen auch in der konnektionistischen Variante begrifflich sein. Das Bild von Wahrnehmungen wird daher insofern von einer Festlegung auf satzartige oder konnektionistische mentale Repräsentationen nicht berührt.

Insgesamt ergibt sich aus Feyerabends und Churchlands Auffassungen zu Messungen und Wahrnehmungen das folgende allgemeine Bild von Beobachtung. Beobachtungsprozesse führen zu Beobachtungssätzen. Im Fall von Wahrnehmungen folgen die Beobachtungssätze auf Empfindungen, bei Messungen auf Zustände des Messgeräts. Die kausale Relation macht sich aber zunächst nur an den syntaktischen Eigenschaften der verursachten Sätze fest. Wodurch die Bedeutung der Sätze festgelegt wird, muss gesondert entschieden werden. Es ist aber denkbar, dass sich die Bedeutung von Sätzen ändert, während die Kausalrelationen bestehen bleiben. Das oben skizzierte Bild soll sich demnach auf alle Beobachtungen anwenden lassen:

Beobachtung: ... Z Zeigerstand/ Empfindung

Beobachtungssatz

Interpretation

Dieses Bild ermöglicht zunächst die Auszeichnung eines besonderen Teils des wissenschaftlichen Vokabulars als Beobachtungsvokabular. Ein Ausdruck gehört demnach für eine wissenschaftliche Gemeinschaft zum Beobachtungsvokabular, wenn er - grob gesagt - in den Beobachtungssätzen vorkommt, die durch Messgeräte oder Beobachter der Gemeinschaft produziert werden. Feyerabend fügt einige zusätzliche Bedingungen an, ${ }^{13}$ aber für unsere Zwecke ist vor allem wichtig, dass Beobachtungsvokabular wie Beobachtungssätze bloß durch kausale Verknüpfungen spezifiziert werden und nicht etwa durch semantische Kriterien (als Ausdrücke, die Inhalte besonderer Art haben).

Aus Feyerabends und Churchlands Bild von Beobachtungen ergeben sich zwei Weisen, auf die Beobachtungen theorieabhängig sein können. Erstens könnten Theorien beeinflussen, welche Sätze überhaupt in der Folge von Empfindungen oder Zuständen des Messgeräts produziert werden. Demnach wäre der Prozess der Produktion der Beobachtungssätze theorieabhängig. Zweitens kann es von Theorien abhängen, welche Bedeutung die Beobachtungssätze haben. ${ }^{14}$

13 Dies sind Einhelligkeit für die Beobachter einer Gemeinschaft, Schnelligkeit der Produktion des Satzes bei ihnen und kausale Abhängigkeit der Produktion von der Situation. Siehe Feyerabend (1958), Abschn. 2.

14 Es ist zusätzlich denkbar, dass Theorien auch beeinflussen, welche Empfindungen man in bestimmten Situationen hat. Feyerabend und Churchland halten eine solche Abhän- 
Es ist sicherlich plausibel, für Messgeräte die erste Form der Theorieabhängigkeit anzunehmen. Messgeräte muss man explizit skalieren, d.h. den Zeigerständen etc. Sätze zuordnen. Und diese Zuordnung wird sicherlich im Lichte all dessen vorgenommen, was man über den Gegenstandsbereich und das Messgerät zu wissen glaubt. Theoretische Annahmen können hierbei eine zentrale Rolle spielen. Insgesamt wird es darum gehen, dem Messgerät Sätze (mit Bedeutungen) so zuzuweisen, dass die Beobachtungen möglichst verlässlich sind. Diese Form der Theoriebeladenheit werde ich im nächsten Kapitel genauer besprechen.

Es ist weniger klar, ob dasselbe auch für Wahrnehmungen gilt. Feyerabend und Churchland gehen davon aus, dass die kausalen Relationen zwischen Empfindungen und Beobachtungssätzen im Wesentlichen aufgrund von Lernprozessen so bestehen, wie sie es tun. Soweit diese Lernprozesse dann vor verschiedenen theoretischen Hintergründen verschieden ablaufen, wäre mit (starker) Theorieabhängigkeit zu rechnen. Die Rede von Sätzen mag dies nahe legen, indem suggeriert wird, dass konventionelle sprachliche Ausdrücke kausal mit Empfindungen verknüpft werden müssen. Dies ist allerdings eine Stelle, an der Vorsicht geboten ist. Denn tatsächlich ist hier von mentalen Zuständen die Rede. Es ist weit weniger klar, dass theorieabhängig erlernt wird, mentale Zustände welcher Struktur in der Folge von welchen Empfindungen produziert werden. Man könnte stattdessen der Ansicht sein, dass die Syntax der Beobachtungssätze bei allen Beobachtern gleich ist, weil sie entweder durch angeborene oder gemeinschafts-invariante Faktoren festgelegt wird..$^{15}$ Die erste Annahme der Theorieabhängigkeit ist daher problematisch. Allerdings wird diese Form möglicher Theorieabhängigkeit im Folgenden nicht weiter von Bedeutung sein.

Stattdessen tritt die zweite mögliche Theorieabhängigkeit in den Vordergrund. Hier geht es nicht darum, welche Syntax produzierte Beobachtungssätze haben, sondern darum, was die Bedeutung dieser Sätze bestimmt. Eine solche Theorieabhängigkeit ergibt sich bei Feyerabend und Churchland, weil sie davon ausgehen, dass erstens der gesamte Inhalt der Beobachtungen in der Form begrifflich ist, in der es auch die Bedeutung von Sätzen ist und zweitens diese begrifflichen Inhalte durch Theorien determiniert sind. Für diese zweite Annahme wird die Netzwerk-Semantik angeführt, auf die ich gleich im

gigkeit zwar für plausibel, räumen ihr aber keinen zentralen Platz in ihrer Argumentation ein. (Siehe Feyerabend 1975, 93/94; Churchland 1988, 185.)

15 So vertritt Fodor die gegenteilige These, dass das mentale Beobachtungsvokabular syntaktisch gesehen nicht in Abhängigkeit vom Lernhintergrund variiert. Siehe Fodor (1984). Für mehr hierzu siehe Kap. 7. 
nächsten Abschnitt zu sprechen komme. Vorher ist abzuschätzen, was für die erste Annahme spricht.

Der Inhalt von Beobachtungen mit Messgeräten ist - soweit keine Bilder produziert werden,$-{ }^{16}$ offensichtlich in sprachtypischer Weise begrifflich. Schließlich führen die betrachteten Messgeräte zu Sätzen im weiten, oben erläuterten Sinn. Die Bedeutung dieser Sätze ist begrifflich und wird durch die verwendeten wissenschaftlichen Ausdrücke bestimmt. Über die Theorieabhängigkeit dieser begrifflichen Inhalte kann daher durch eine Semantik für die fraglichen sprachlichen Ausdrücke entschieden werden.

Wiederum ist es aber unklar, ob der Fall der Wahrnehmung zu dem der Messung analog ist. Zwar sprechen Feyerabend und Churchland auch hier von Beobachtungssätzen. Damit legen sie nahe, dass die Inhalte wie bei gewöhnlichen Sätzen begrifflich sind und gebräuchliche semantische Theorien die Festlegung der Inhalte beschreiben. Allerdings wurde schon festgestellt, dass damit insbesondere Zustände perzeptuellen Erscheinens, wie etwa das Aussehen von etwas wie ein Lindenbaum, beschrieben werden. Zunächst könnte man hier für die Annahme argumentieren, dass solche Wahrnehmungsinhalte gar nicht begrifflich sind. Wie im vorigen Kapitel schon gesagt, werde ich aber nicht versuchen, eine solche Position gegen die Theoriebeladenheit stark zu machen, sondern vielmehr davon ausgehen, dass Zustände des perzeptuellen Erscheinens als $F$ schon eine perzeptuelle Klassifikation als $F$ einschließen. Aber auch dann bleibt die Frage, begriffliche Fähigkeiten welcher Art für eine solche perzeptuelle Klassifikation notwendig sind. Perzeptuelle Inhalte setzen plausiblerweise keine Sprache voraus. Daher ist zu erwarten, dass für sprachliche begriffliche Inhalte andere Anforderungen bestehen als für perzeptuelle. Insbesondere könnte man glauben, dass Klassifikationsfähigkeiten, die perzeptuelle begriffliche Inhalte ermöglichen, allein durch Wahrnehmung erworben werden können, wohingegen sprachliche Inhalte kompetente Sprecher voraussetzen.

Indem Feyerabend und Churchland den gesamten Inhalt von Wahrnehmungen als den von Beobachtungssätzen beschreiben, übergehen sie diese Möglichkeit einer Differenzierung zwischen sprachlichen Fähigkeiten und perzeptuellen Fähigkeiten. Darin liegt eine Voraussetzung, die für ihre Position wichtig ist. Denn nur so lässt sich mit einer allgemeinen semantischen Theorie für die Theorieabhängigkeit aller Beobachtungsinhalte argumentieren. Diese Voraussetzung werde ich um dieser Argumentation willen für dieses Kapitel zugestehen. Erst in Kapitel 7 werde ich sie angreifen und einige

16 Zu bildgebenden Verfahren siehe Kap. 7, Abschn. 6. 
Besonderheiten perzeptueller begrifflicher Fähigkeiten und ihres Erwerbs herausarbeiten.

Damit komme ich jetzt dazu, die zweite, in der Einleitung zu diesem Kapitel angekündigte Annahme zu diskutieren, die zur FeyerabendChurchland'schen Form der Theoriebeladenheit führt: die Annahme der Netzwerk-Semantik.

\section{Eine Semantik für Beobachtungsterme}

\section{a) Die Netzwerk-Semantik}

Feyerabend und Churchland vertreten eine semantische Theorie für Beobachtungsausdrücke, die man als ,Netzwerk-Semantik ${ }^{6}$ bezeichnen kann. ${ }^{17}$ Damit ist eine Spielart einer Begrifflichen-Rollen-Semantik gemeint. Der Theorie zufolge wird die Bedeutung eines Beobachtungsausdrucks vor allem durch das Netz allgemeiner Überzeugungen oder Hypothesen festgelegt, in dem er vorkommt. Für diese Grundannahme gibt es eine alternative Formulierung. Allgemeine Sätze der Form, Wenn etwas $F$ ist, dann ist es auch $G^{6}$ rechtfertigen einen Schluss von einem $F$ auf ein $G$. Der Grundidee der Netzwerk-Semantik zufolge wird daher die Bedeutung eines Beobachtungsausdrucks auch durch das Muster der gerechtfertigten Schlüsse festgelegt, in denen ein Ausdruck vorkommt.

Diese Grundidee lässt sich in verschiedenen Hinsichten weiter präzisieren. Erstens kann man spezifizieren, welche Schlüsse oder Sätze die Bedeutung des Ausdrucks festlegen und damit semantisch konstitutiv sein sollen. Klassischerweise sollten dies nur die analytischen Schlüsse sein. Stattdessen könnte man auch einige oder alle materialen Schlüsse für semantisch bestimmend halten. Feyerabend und Churchland sind skeptisch darüber, dass sinnvoll zwischen analytischen und synthetischen Schlüssen oder Sätzen unterschieden werden kann. Sie betrachten daher auch materiale Schlüsse bzw. Sätze als semantisch konstitutiv. Churchland vertritt zudem die Ansicht, dass verschiedenen Sätzen dabei ein unterschiedliches Gewicht zukommt. Einige Prinzipien seien wichtiger für die Bestimmung der Bedeutung eines Ausdrucks, andere dagegen leisteten nur einen geringen Beitrag. Aber auch die zentraleren Annahmen seien nicht analytisch, sondern könnten revidiert werden. Zudem lässt Churchland die Möglichkeit zu, dass auch singuläre Sätze, und hierbei auch solche mit indexikalischen Ausdrücken, zum semantisch be-

17 Churchland spricht vom ,network approach“ (Churchland 1988, 181), während Feyerabend oft von der „contextual theory of meaning“ spricht (Feyerabend 1962, 68). 
stimmenden Netzwerk gehören. Churchland denkt hierbei insbesondere an solche Sätze wie ,Wasser ist der Stoff in unseren Seen und Flüssen'.18

Zweitens kann man präzisieren, in welchem Umfang die Bedeutung oder allgemeiner die semantischen Eigenschaften von Beobachtungsausdrücken durch das Netzwerk festgelegt werden. Feyerabend sowie an vielen Stellen auch Churchland sehen die Bedeutung eines Ausdrucks vollständig durch das Netzwerk festgelegt. ${ }^{19}$ Aber bisweilen zieht sich Churchland auch auf die schwächere Position zurück, dass der Einfluss des Netzwerks durch kausale Faktoren oder Umstände der Umwelt beschränkt ist. ${ }^{20}$ Auch hierbei wird der Einfluss des Netzes jedoch immer als dominierend angenommen, so dass ein und derselbe Ausdruck völlig verschiedene und unvereinbare Bedeutungen haben kann in Abhängigkeit davon, in welches Netz er eingebettet ist.

In jüngerer Zeit hat Churchland eine alternative Formulierung dieser Netzwerk-Semantik gegeben. Hierbei stützt er sich auf eine konnektionistische Auffassung mentaler Repräsentationen und kognitiver Verarbeitung. Der Konnektionismus fasst mentale Repräsentationen nicht als satzartige Einzeldinge auf, die gemäß explizit repräsentierten Schlussregeln verarbeitet werden. Vielmehr werden Zustände neuronaler Netze, und hierbei in der Regel die Muster der Aktivation einer Ebene von Einheiten (,Neuronen'), als mentale Repräsentationen betrachtet. Die Verarbeitung dieser Repräsentationen erfolgt dann nicht durch einen zentralen Prozessor, sondern verteilt entlang den Verbindungen zur nächsten Ebene von Einheiten. Das Muster der Aktivation einer Ebene ist insgesamt durch die Aktivation der vorhergehenden Ebene, die Art der Verschaltung, die Gewichtung der Verbindungen sowie die Summationsfunktion (die Funktion, die der Summe des Inputs einer Einheit eine Aktivation der Einheit zuordnet), festgelegt. ${ }^{21}$

In der Churchland'schen Netzwerk-Semantik für konnektionistische mentale Repräsentationen können nicht mehr Schlussregeln zwischen Sätzen semantisch konstitutiv sein. Mentale Repräsentationen sind dem Konnektionismus zufolge nicht satzartig und werden nicht gemäß Schlussregeln verarbeitet. Stattdessen werden die Relationen zu anderen Aktivationsmustern, zu denen ein Aktivationsmuster gemäß der Struktur des Netzes und der Gewichtung seiner Verbindungen steht, als konstitutiv für dessen Inhalt angese-

18 Siehe etwa Churchland (1979), Kap. 3. Um Churchlands semantischen Gradualismus hat sich eine Debatte mit Fodor und Lepore entwickelt. Siehe die Stellungnahmen in McCauley (1996) sowie Churchland (1998) und Fodor/Lepore (1999).

19 Siehe etwa Feyerabend (1981), x; Churchland (1979), 14.

20 Siehe Churchland (1985a), 287; (1988), 183; (1998).

21 Eine einleitende Darstellung neuronaler Netze gibt Churchland (1990). Vgl. auch z.B. Bechtel/ Abrahamsen (1991). 
hen. Für ein bestimmtes Muster einer Ebene sollen insbesondere die Aktiviationsmuster vorhergehender Ebenen, durch die es hervorgebracht werden kann, und die Aktiviationsmuster der nachfolgenden Ebenen, die es hervorbringt, semantisch bestimmend sein. ${ }^{22}$

Da aber die Inhalte der konnektionistischen mentalen Repräsentationen als begrifflich angenommen werden, ergibt sich ihr Inhalt weiterhin aus der Rolle, die sie in der kognitiven Verarbeitung begrifflich gehaltvoller Zustände spielen. Auch die konnektionistische Variante der Netzwerk-Semantik ist eine Begriffliche-Rollen-Semantik. ${ }^{23}$

Aus der Netzwerk-Semantik ergibt sich sehr unmittelbar, dass die Bedeutung von Beobachtungsausdrücken von Theorien abhängt. Man kann hierzu grob zwischen zwei verschiedenen Konzeptionen von Theorien unterscheiden. In einem schwachen Sinn gilt jedes Netzwerk von Begriffen als Theorie, da ein solches Netzwerk eine rudimentäre Taxonomie für einen Gegenstandsbereich einschließt. ${ }^{24}$ Dann sind Ausdrücke wie ,grün“ oder ,Hund“ schon theorieabhängig, da sie in einem Netzwerk von Begriffen stehen, das im schwachen Sinn theoretisch ist (Farbausdrücke oder Ausdrücke für sinnliche Qualitäten im einen, Tier- oder Haustierausdrücke im anderen Fall).

Ein stärkerer Begriff von Theorie würde von einem theoretischen Netzwerk verlangen, dass es eine größere Zahl von Phänomenen umfasst und ihnen gegenüber Erklärungskraft besitzt. ${ }^{25}$ Typischerweise müssen hierfür Entitäten und Mechanismen postuliert werden, die nicht direkt wahrnehmbar

22 Siehe Churchland/ Churchland (1996), 274-77.

23 Wenn man die Churchland'schen Äußerungen zur Semantik konnektionistischer mentaler Repräsentationen genau betrachtet, kann man (mindestens) drei Stufen der Position unterscheiden (vgl. hierzu Tiffany 1999). Die von mir dargestellte Variante, die am deutlichsten eine Begriffliche-Rollen-Semantik darstellt, macht Stufe zwei aus.

Stufe eins sieht den Inhalt eines Aktivationsmusters durch sog. ,microfeatures festgelegt, d.h. durch spezifische Inhalte, die die Aktivationsgrade einzelner Einheiten haben, aus denen das Muster besteht. Es ist klar, dass diese Mikromerkmals-Position eigentlich keine vollständige philosophische Semantik ist, da darin lediglich der Inhalt komplexer Zustände zu Inhalten der Teile des Zustands in Beziehung gesetzt wird. Für Kritik an dieser Position siehe Fodor/ Lepore (1996), und für eine weitgehende Rücknahme der Position Churchland/ Churchland (1996).

In Churchland (1998) hat Churchland eine dritte Stufe angedeutet, wonach nicht die Relationen zu vorhergehenden und nachfolgenden Aktivationsmustern, sondern die Relationen zu alternativen Mustern derselben Ebene sowie zu externen Ursachen dieser Muster semantisch konstitutiv sein sollen. Einen vergleichbaren Vorschlag hat Tiffany (1999) skizziert. Es ist aber unklar, ob diese Position noch eine BegrifflicheRollen-Semantik darstellt und wie sie sich zur Variante der Theoriebeladenheit verhält, die Thema dieses Kapitels ist. Für Kritik der dritten Stufe siehe Fodor/ Lepore (1999).

24 Siehe Churchland (1988), 182.

25 Vgl. Churchland (1979), \$3. 
sind. Aber die Bedeutung von Beobachtungssätzen ist der Netzwerk-Semantik zufolge auch von Theorien in diesem Sinn abhängig. Dies gilt jedenfalls für Beobachtungssätze, die potenziell für Theorien evidenziell relevant sind. Denn damit Beobachtungssätze als Evidenz für oder gegen Theorien sprechen können, müssen sie mit diesen in inferenziellen Beziehungen stehen. Die Beobachtungssätze müssen beispielsweise aus den Theorien ableitbar sein. Dann gehören aber auch die Theorien, für die ein Beobachtungssatz potenziell evidenziell relevant ist, zum Netzwerk von Sätzen, das die Bedeutung der Beobachtungsausdrücke festlegt. ${ }^{26}$

Feyerabend und Churchland vertreten die Netzwerk-Semantik nicht nur für Beobachtungsausdrücke, sondern auch für alle anderen, insbesondere theoretischen wissenschaftlichen Ausdrücke. Ihre Semantik ist demnach holistisch. Ihnen zufolge erhält generell eine Menge von Ausdrücken Bedeutung durch das Geflecht inferenzieller Relationen, in dem die Ausdrücke zueinander stehen. Keiner der Ausdrücke ist unabhängig von diesem Netz bedeutungsvoll, vielmehr wird die Bedeutung jedes Ausdrucks durch seine Position im Netz bestimmt. Veränderungen in diesem Netz haben daher Auswirkungen auf die Bedeutung aller verbundenen Ausdrücke.

Was spricht für diese Netzwerk-Theorie als Semantik speziell für Beobachtungsausdrücke? Bei Feyerabend und Churchland findet sich eine große Zahl von Überlegungen. Sie lassen sich aber weitgehend in zwei Argumentationslinien zusammenfassen. Erstens argumentieren sie dafür, dass die Netzwerk-Theorie eine angemessene Semantik für theoretische Ausdrücke darstellt und dass es zwischen solchen theoretischen Ausdrücken und Beobachtungsausdrücken keine wesentlichen semantischen Unterschiede gibt. Ich nenne dies das Argument von der Semantik theoretischer Ausdrücke. Zweitens weisen sie einige alternative semantische Theorien für Beobachtungsausdrücke zurück und schließen so auf die Netzwerk-Theorie als einzige verbleibende Option. Ich nenne dies das Ausschluss-Argument. Die beiden Argumente ergänzen sich ihrer Struktur nach sehr gut, so dass sie zusammen genommen, sofern die Prämissen haltbar sind, eine starke Begründung der NetzwerkTheorie bilden.

Churchland scheint zu glauben, dass sich zusätzliche Gründe für die Netzwerk-Semantik und damit die Theorieabhängigkeit aus der konnektionistischen Auffassung mentaler Repräsentationen ergeben. ${ }^{27}$ Dies ist aber sehr unwahrscheinlich. Erstens ist der Konnektionismus selbst sehr umstritten. So

${ }_{26}$ Vgl. Feyerabend (1958), 18, seine „These 1“. Feyerabend vertritt auch die weitergehende These, dass der semantische Gehalt einer Theorie auch von den in Betracht gezogenen theoretischen Alternativen abhängt. Siehe Feyerabend (1962), 68/69.

27 Siehe etwa Churchland (1990) und (1992). 
wird er mit dem Einwand konfrontiert, dass er die Systematizität und Produktivität des Denkens nicht erklären kann und daher als allgemeine Theorie mentaler Repräsentation und kognitiver Verarbeitung scheitert. ${ }^{28}$ Zwar kann der Konnektionismus beachtliche Ergebnisse etwa in der Simulation des perzeptuellen Erkennens von Mustern aufweisen. ${ }^{29}$ Wenn man aber nur Teile der kognitiven Architektur wie perzeptuelles Erkennen konnektionistisch auffasst, lässt sich damit die Theoriebeladenheit nicht mehr begründen. Denn diese Begründung muss darauf bauen, dass auch zentrale theoretische Kompetenzen konnektionistisch aufgebaut sind. Andernfalls gehören unsere theoretischen Überzeugungen nicht zum bedeutungsbestimmenden Netzwerk.

Zweitens ist der Konnektionismus vor allem eine Theorie mentaler Repräsentationen und kognitiver Verarbeitung, d.h. es geht darum, wie die mentalen Träger semantischer Gehalte strukturiert und verarbeitet werden. Zur Diskussion steht, ob diese Träger satzartig sind oder als Aktivationsmuster aufgefasst werden, ob sie durch einen zentralen Prozessor nach expliziten Regeln oder verteilt nach vom Netz verkörperten Funktionen verarbeitet werden. Wie diese Repräsentationen zu ihren Inhalten kommen, ist eine davon völlig unabhängige Frage. Mit dem Konnektionismus sind ganz verschiedene Positionen der Semantik vereinbar, etwa externalistische (z.B. kausale), oder eben auch eine Begriffliche-Rollen-Semantik. ${ }^{30}$ Selbst wenn der Konnektionismus daher als allgemeine Theorie der Kognition gut begründet wäre, würde dies nicht ausdrücklich zugunsten der Netzwerk-Semantik sprechen. Ich werde daher nur das Argument von der Semantik theoretischer Ausdrücke und das Ausschluss-Argument diskutieren.

\section{b) Das Argument von der Semantik, theoretischer Ausdrücke}

Das Argument von der Semantik theoretischer Ausdrücke hat zwei Prämissen. Erstens wird behauptet, dass die Bedeutung theoretischer Ausdrücke durch das Netzwerk allgemeiner Sätze festgelegt wird, in denen sie vorkommen. Zweitens wird dafür argumentiert, dass es zwischen theoretischen Ausdrücken und Beobachtungstermen keine semantischen Unterschiede gibt. Daraus folgt offensichtlich, dass auch die Bedeutung von Beobachtungstermen durch das Netzwerk von Sätzen bestimmt wird. Bevor das Für und Wider der Prämissen bewertbar ist, muss die Theorie-Beobachtung-Unterscheidung, auf die hier Bezug genommen wird, geklärt werden.

\footnotetext{
28 Siehe Fodor/ Pylyshyn (1988).

29 Siehe Bechtel/ Abrahamsen (1991), Kap. 4.

30 Dieser Punkt wird auch von Tiffany (1999) und Fodor/ Lepore (1999) herausgestellt.
} 
(i) Die Theorie-Beobachtung-Unterscheidung

Traditionellerweise werden theoretische Ausdrücke den Beobachtungsausdrücken gegenübergestellt. Die logisch-empiristische Standardsicht wissenschaftlicher Theorien ging davon aus, dass es - von logischen und mathematischen Ausdrücken abgesehen - eine ausschließende, erschöpfende und universelle Unterteilung des wissenschaftlichen Vokabulars in theoretische Terme und Beobachtungsausdrücke gibt. Wie jedoch seither oft festgestellt wurde, sind hierbei nicht eine, sondern zwei Unterscheidungen am Werk. ${ }^{31}$ Zum einen kann man zwischen Beobachtbarem und Unbeobachtbarem unterscheiden, zum anderen können Ausdrücke theoretisch oder nicht theoretisch sein.

Die Unterscheidung, beobachtbar - nicht beobachtbar' betrifft primär Dinge (Gegenstände, Ereignisse, Eigenschaften). Erst abgeleitet davon kann man Ausdrücke danach unterscheiden, ob sie Beobachtbares oder Unbeobachtbares bezeichnen. Man kann hierbei unterschiedlich weite Begriffe der Beobachtbarkeit vertreten, nach denen der Bereich des Beobachtbaren verschieden umfangreich ist. Man könnte nur Dinge für beobachtbar halten, die von uns ohne Hilfe von Instrumenten wahrgenommen oder erkannt werden können. Demnach wären Jupitermonde, nicht aber Bakterien beobachtbar. Oder man kann auch solche Objekte hinzunehmen, die man nur mit Hilfe von Instrumenten wahrnehmen kann, wodurch Bakterien und anderes Kleines beobachtbar wären. Elektronen und ihr Spin, die starke Wechselwirkung oder chemische Bindungen wären demnach nicht beobachtbar. Und schließlich kann man alles, was mit Instrumenten und Experimenten überhaupt detektierbar ist, als beobachtbar bezeichnen, ohne dass Wahrnehmung hierbei eine ausgezeichnete Rolle zu spielen bräuchte. Danach wären selbst Neutrinos beobachtbar. ${ }^{32}$

$\mathrm{Da}$ ich an Beobachtungen vor allem insoweit interessiert bin, als damit empirische Beiträge zur wissenschaftlichen Erkenntnisgewinnung beschrieben werden, folge ich in dieser Studie dem weitesten der Begriffe und bezeichne dasjenige als beobachtbar, mit dem man epistemisch fruchtbar interagieren kann. Allerdings wird sich aus der Diskussion der Theoriebeladenheit von Beobachtungen ergeben, dass es eine epistemisch wichtige Untergruppe von Beobachtungen gibt, die ungefähr vom Beobachtungsbegriff mittleren Umfangs erfasst wird. Demnach will ich alles, was auf eine Weise beobacht-

31 Siehe etwa Putnam (1962); van Fraassen (1980), 14; Kosso (1989), 12/13.

32 Für Beobachtungsbegriffe ungefähr entsprechend der drei genannten Umfänge siehe jeweils van Fraassen (1980), Hacking (1983) und Shapere (1982). Siehe auch Feyerabend (1960), 42/43 für den weitesten Beobachtungsbegriff. 
bar ist, bei der perzeptuelle Fähigkeiten eine zentrale Rolle spielen, als perzeptuell beobachtbar bezeichnen. Für die Zwecke des Arguments von der Semantik theoretischer Ausdrücke werden am besten Ausdrücke für perzeptuell beobachtbare Dinge betrachtet. ${ }^{33}$

Die Unterscheidung in theoretisch und nicht theoretisch betrifft primär Ausdrücke. Ein Ausdruck könnte demnach als theoretisch gelten, wenn er wesentlich in einer Theorie vorkommt oder durch eine solche eingeführt wird. Wie eben schon ausgeführt, gibt es auch hier unterschiedlich weite Begriffe der Theorie. Allerdings ist der schwache Begriff, demzufolge schon eine Menge aufeinander bezogener Begriffe eine Theorie darstellt, für die gegenwärtige Diskussion nicht brauchbar, da diesem Begriff zufolge fast alle Ausdrücke, wie auch ,grün' oder ,Hund', theoretisch sind. Besser geeignet ist hier ein anspruchsvollerer Begriff von Theorie, demzufolge eine Theorie ihrer Art nach explanatorische Kraft gegenüber einem großen und in gewissem Ausmaß disparaten Phänomenbereich besitzen sollte. In Ableitung davon kann man dann auch Dinge der Welt als theoretisch bezeichnen, indem man damit meint, dass sie überwiegend von theoretischen Ausdrücken bezeichnet werden oder ihre Existenz theoretisch postuliert wird.

Wenn man die beiden Unterscheidungen so auseinander hält, gibt es Dinge, die sowohl theoretisch postuliert als auch beobachtbar sind. Dies ist offensichtlich für den Fall, dass man den weitesten Begriff von Beobachtungen voraussetzt, gilt aber auch für perzeptuell beobachtbare Gegenstände. So wurden beispielsweise Träger und Mechanismen der biologischen Vererbung oder Infektionskeime zuerst theoretisch angenommen und dann mikroskopisch beobachtbar.

Allerdings kommt es sehr häufig vor, dass theoretisch postulierte Dinge nicht perzeptuell beobachtbar sind. Dies gilt etwa für die oben aufgezählten, nicht perzeptuell beobachtbaren Entitäten. Es scheint, dass dies kein Zufall ist. Entitäten müssen gerade dann theoretisch postuliert werden, und die Einführung von Ausdrücken dafür muss gerade dann über explanatorische Theorien erfolgen, wenn man keinen unabhängigen Zugang zu Instanzen der Dinge durch die Wahrnehmung hat (und sei es durch Wahrnehmung mit Hilfe von Instrumenten). Zwar kommt es vor, dass unsere Möglichkeiten der Wahrnehmung sich verbessern, indem wir neue Bereiche der Welt erschließen oder über neue Instrumente verfügen, so dass zuvor bloß theoretisch postulierte Entitäten perzeptuell beobachtbar werden. (Die angeführten Beispiele für theoretisch postulierte und gleichwohl perzeptuell beobachtbare Entitäten

33 Für weitere Überlegungen zur Beobachtbarkeit siehe das Kapitel 6. 
sind gerade von dieser Art.) Dennoch kommt der Fall regelmäßig vor, dass theoretische Ausdrücke nichts perzeptuell Beobachtbares beschreiben.

Für die Zwecke des Arguments von der Semantik theoretischer Ausdrücke ist es sinnvoll, sich auf die so verstandene Unterscheidung zwischen theoretischen Ausdrücken und Beobachtungsausdrücken zu stützen. Demnach behauptet die erste Prämisse, dass eine Netzwerk-Semantik für theoretische Ausdrücke, die nichts gegenwärtig perzeptuell Beobachtbares beschreiben, angemessen ist. Die zweite Prämisse behauptet dann, dass es keine semantischen Unterschiede zwischen solchen Ausdrücken und Ausdrücken für perzeptuell Beobachtbares gibt.

(ii) Theoretische Ausdrücke

Hiermit komme ich zur ersten Prämisse. Für theoretische Ausdrücke, die nichts perzeptuell Beobachtbares bezeichnen, besitzt eine Netzwerk-Semantik einige Anfangsplausibilität. An einem Beispiel Churchlands lässt sich diese Plausibilität aufzeigen. Angenommen, wir führen den Term ,Phlogiston“ durch eine einfache Theorie mit den folgenden vier Annahmen ein:

(1) Phlogiston ist eine elementare Substanz.

(2) Phlogiston bildet Verbindungen mit anderen Substanzen.

(3) Verbrennen und Kalzinieren bestehen im Freisetzen von Phlogiston aus einer Verbindung, die es enthält.

(4) Das Freisetzen von Phlogiston wird von hohen Temperaturen hervorgerufen. ${ }^{34}$

Churchland schreibt:

Let us put ourselves in the position of a proto-chemist who has just formulated this theory in hopes of rendering intelligible the details of certain familiar physical transformations. Aside from a few leading assumptions as to which substances are phlogiston compounds (wood, metals), the set of assumptions (1)-(4) effectively exhausts our conception of phlogiston, our understanding of the term ,phlogiston'. These are the sentences that introduce the term into our general linguistic commerce, and it has no source of semantic identity, initially at least, beyond these. (Churchland 1979, 47; Churchlands Hervorhebung.)

Im Rahmen dieses Beispiels ist es plausibel anzunehmen, dass unser Verständnis des Ausdrucks von der Theorie (vielleicht zusammen mit den intendierten Anwendungsfällen) festgelegt wird. Ohne die Theorie hätte der Ausdruck keine Bedeutung, und mit einer anderen Theorie hätte er eine

\footnotetext{
34 Siehe Churchland (1979), 47.
} 
andere. Wer demnach ,Phlogiston' vollständig verstehen will, muss die Theorie kennen. Und die Wahrheitsbedingungen von Sätzen, in denen der Ausdruck ,Phlogiston' vorkommt, etwa von Behauptungen wie ,da entweicht Phlogiston', werden durch die Theorie bestimmt. Demnach wären erstens dasjenige, was man zum Verständnis des Ausdrucks kennen muss, und zweitens die Wahrheitsbedingungen von Sätzen mit dem Ausdruck durch das theoretische Netzwerk bestimmt.

Allerdings sind diese Behauptungen für theoretische Terme in Frage gestellt worden. Insbesondere Putnam hat, in der Folge von Kripke, eine Semantik unter anderem für theoretische Terme vorgeschlagen, wonach erstens Referenz und Intension des Ausdrucks nicht durch Theorien, sondern weitgehend durch die Welt festgelegt werden, und zweitens auch das Verständnis des Ausdrucks oder semantische Kompetenz für den Ausdruck nicht das Kennen einer Theorie voraussetzen. ${ }^{35}$

Die Kernidee besteht darin, dass die Referenz eines theoretischen Ausdrucks festgelegt werden kann, indem man bei der Einführung des Ausdrucks auf irgendeine Weise ein Exemplar der Art, die der Ausdruck bezeichnen soll, herausgreift. Dieses Herausgreifen kann mit Hilfe einer Beschreibung geschehen, die bloß kontingenterweise (nicht semantisch notwendig) auf das Exemplar zutrifft, oder es kann im Extremfall auch rein demonstrativ sein. Zum Beispiel kann man den Ausdruck, Wasser' (der allerdings kein theoretischer Ausdruck ist) einführen, indem man bestimmt, dass er das Zeug in unseren Seen und Flüssen bezeichnen soll. Oder man kann auf eine Menge Wasser deuten und sagen: Dieses Zeug ist Wasser.

Das bloße Herausgreifen eines Exemplars der Art kann hinreichend für die Festlegung einer Referenz (einer Extension in der tatsächlichen Welt) und sogar einer Intension (einer Extension in jeder möglichen Welt) sein, sofern man einen starken Arten-Realismus voraussetzt. Diesem Realismus zufolge gehört erstens aufgrund der Beschaffenheit der tatsächlichen Welt ein herausgegriffenes Exemplar zu einer bevorzugten Klasse von Dingen, die damit als Extension des Ausdrucks bestimmt werden kann. Indem man auf eine vorliegende Menge von Wasser deutet, muss demnach klar sein, welches Zeug in unserer Welt damit exemplarisch herausgegriffen wird. Zweitens soll damit eine Extension in allen möglichen Welten festgelegt sein, indem das herausgegriffene Exemplar essenzielle Eigenschaften haben soll, anhand derer sich die Zugehörigkeit zur herausgegriffenen Art auch in anderen möglichen Welten bestimmt. Demnach bezeichnet unser Ausdruck, Wasser ${ }^{6}$ nur Substanzen, die dieselbe chemische Zusammensetzung wie unser Wasser (das

35 Siehe Putnam (1973) und (1975a). 
Wasser in der tatsächlichen Welt) haben. In dem Maß, in dem man auf die Annahme eines solchen Arten-Realismus verzichtet, kann nicht die Welt selbst die nötigen eindeutigen Ähnlichkeitsrelationen zwischen dem Exemplar und der Extension in unserer Welt und in allen möglichen Welten zur Verfügung stellen. Dann muss man diese Relationen durch weitere explizite Beschreibungen oder durch andere beteiligte Umstände spezifizieren.

Wenn auf diese Weise eine Art herausgegriffen und ein Ausdruck dabei als Bezeichner für diese Art erfolgreich in die Sprache eingeführt wird, dann hat dieser Ausdruck eine Bedeutung: Er hat eine Art als Intension sowie die tatsächlichen Exemplare der Art als Extension. Damit ist sein Beitrag zu den Wahrheitsbedingungen der Sätze, in denen er vorkommt, festgelegt (soweit keine hyper-intensionalen Kontexte betrachtet werden). Eine Theorie braucht hierbei keine wesentliche Rolle zu spielen. Alle Mitglieder der Sprachgemeinschaft können dann unabhängig von Theorien mit dem Ausdruck auf die Art Bezug nehmen und Behauptungen über die Art aufstellen.

Zudem soll für linguistische Kompetenz und das Verstehen von Ausdrücken viel weniger Wissen erforderlich sein als angenommen. Erstens betont Putnam die Möglichkeit linguistischer Arbeitsteilung. Damit ich einen Ausdruck kompetent verwenden kann, muss ich nicht selbst über Wissen verfügen, das dem Wissen einer Theorie über die Dinge irgendwie nahe kommt. Es ist hinreichend für Kompetenz, dass es Experten für die Dinge gibt, die eine solche Theorie kennen, und ich in geeigneter Relation zu diesen Experten stehe (indem ich beispielsweise den Ausdruck von ihnen gelernt habe oder ihn mit einer auf sie bezogenen Absicht verwende). Allerdings ist die Möglichkeit linguistischer Arbeitsteilung nicht an eine Putnam'sche Semantik geknüpft. Vielmehr kann auch Churchland weitgehend davon ausgehen. Denn man könnte immer noch festhalten, dass semantische Kompetenz sich daran festmacht, dass wenigstens die Experten über eine Theorie verfügen, durch die das Verständnis des Ausdrucks festgelegt wird. ${ }^{36}$ Aber die zweite Möglichkeit der Reduzierung notwendigen Wissens ergibt sich erst vor dem Hintergrund der Putnam'schen Weise der Festlegung der Bedeutung. Indem ein Ausdruck schon durch das Herausgreifen eines Exemplars eine Intension erhält, haben Sätze mit diesem Ausdruck Wahrheitsbedingungen unabhängig davon, ob es überhaupt eine Theorie für diese Ausdrücke gibt, und auch unabhängig davon, wie eine eventuell bestehende Theorie beschaffen ist. Ich muss dann für solche linguistische Kompetenz nur in geeigneter Relation zu dieser Einführung des Ausdrucks stehen. Demnach braucht es in der Sprachgemeinschaft nur sehr wenig Wissen über die bezeichneten Entitäten zu

36 Siehe Churchland (1979), $\$ 8$. 
geben, etwa nur Wissen über typische Instanzen und die grobe Art von Dingen, um die es sich handelt. Auch sprachliche Kompetenz für die Ausdrücke wäre demnach einerseits weitgehend ohne theoretische Annahmen möglich, andererseits könnte sie auch von theoretischen Annahmen, wenn es sie gibt, unabhängig bleiben.

Allerdings lassen sich erhebliche Zweifel daran anmelden, dass diese semantische Theorie für theoretische Ausdrücke, die nichts perzeptuell Beobachtbares bezeichnen, in der vorgestellten Weise funktionieren kann. Zunächst ist es für solche Ausdrücke nicht möglich, Exemplare der Referenz rein demonstrativ herauszugreifen. Denn es wird gerade angenommen, dass die Exemplare nicht perzeptuell beobachtbar sind. Man kann daher nicht auf sie deuten. Die Exemplare müssen daher wohl mit Hilfe einer Beschreibung herausgegriffen werden. In einem sehr einfachen Fall könnte diese Beschreibung von der Form , die Ursache von dem' haben, wobei man auf einen perzeptuell beobachtbaren Umstand zeigt. Aber häufig wird es eine solche einfache Beschreibung nicht geben. Erstens haben so gut wie alle Umstände sehr viele Ursachen. Man müsste daher weitere Spezifikationen hinzufügen, um die richtige Ursache festzulegen. Zweitens sind theoretische Entitäten häufig nicht bloß sozusagen einen einzigen kausalen Schritt vom perzeptuell Beobachtbaren entfernt. Für einen Ausdruck wie ,starke Wechselwirkung، findet man unter Umständen keine perzeptuell beobachtbare direkte Wirkung. Auf die Stabilität von Atomkernen kann man ebenso wenig unmittelbar zeigen wie auf die starke Wechselwirkung selbst. Man muss dann aber den theoretischen Term wohl mit Hilfe einer Beschreibung einführen, und in dieser werden sowohl andere theoretische Terme wie auch angenommene Relationen zwischen diesen Termen auftreten. Dann hängt die Referenzfixierung doch wieder von theoretischem Wissen ab. Zudem droht ein Regress: Man kann die Bedeutung des theoretischen Ausdrucks nur fixieren, wenn die hierfür benutzen theoretischen Ausdrücke schon eine Bedeutung haben. Wie ist aber diese festgelegt?

Beispiele wie ,starke Wechselwirkung' oder ,Neutrino' legen zudem einen weiteren Mangel in einer Putnam'schen Semantik für theoretische Ausdrücke offen. Theoretische Begriffe für nicht perzeptuell Beobachtbares sind typischerweise über postulierte kausale Relationen mit sehr vielen perzeptuell beobachtbaren Phänomenen verknüpft. Dies scheint kein Zufall zu sein. Denn es ist gerade der Witz des Postulierens nicht perzeptuell beobachtbarer Entitäten, dass man dadurch eine umfassende Erklärung einer großen und disparaten Menge von Phänomenen erreicht. Ohne dieses Ziel hätte man keinen Grund, die Existenz der Entitäten überhaupt anzunehmen. Daher wird verständlich, dass solche theoretischen Begriffe typischerweise tatsäch- 
lich nicht demonstrativ oder mit Hilfe einer Beschreibung anhand eines einzelnen Phänomens eingeführt werden, sondern im Rahmen theoretischer Erwägungen. ${ }^{37}$ Man erhofft sich von der Einführung die Möglichkeit der Erklärung vieler, disparater Phänomene. Es wäre irrational, die Bedeutung des Ausdrucks von einer einzelnen Relation zu einem Phänomen abhängig zu machen. Vielmehr könnten solche Relationen höchstens einen partiellen Beitrag zur Bedeutungsfixierung leisten. ${ }^{38} \mathrm{Da}$ die explanatorische Fruchtbarkeit eines theoretischen Ausdrucks an der Rolle hängt, den dieser Ausdruck innerhalb der ganzen Theorie spielt, trägt diese Rolle plausiblerweise aber die Hauptbürde in der Bedeutungsfestlegung. Dass wir bei theoretischen Ausdrücken für nicht perzeptuell Beobachtbares dem auch folgen, wird daran deutlich, dass wir typischerweise nicht die Fähigkeit der Erklärung eines besonderen, einzelnen Phänomens, sondern den Beitrag zur Erklärungskraft gegenüber einem großen Phänomenbereich zur Bedingung dafür machen, dass man von der tatsächlichen Existenz der postulierten Entität ausgehen kann.

(iii) Beobachtungsausdrücke

Aber auch wenn dies bedeutet, dass einiges zugunsten einer NetzwerkSemantik für theoretische Terme, die nichts perzeptuell Beobachtbares bezeichnen, spricht, ist noch offen, wie es um die zweite Prämisse des Arguments von der Semantik theoretischer Ausdrücke steht. Gibt es Gründe dafür anzunehmen, dass Ausdrücke für perzeptuell Beobachtbares semantisch auf gleiche Weise funktionieren?

Churchland hat eine interessante Fiktion einer menschlichen Gemeinschaft entworfen, bei der es zunächst sehr plausibel erscheint anzunehmen, dass Ausdrücke, die semantisch wesentlich durch eine Rolle in einer Theorie bestimmt sind, für Wahrnehmbares verwendet werden. Die ,Freunde des Wärmestoffs' verfügen über ein alltägliches Temperaturvokabular und alltägliche allgemeine Annahmen über Temperatur, die ungefähr denen der Wärmestoff-Theorie des 19. Jahrhunderts entsprechen. ${ }^{39}$

Die Wärmestoff-Freunde machen eine ganze Reihe allgemeiner (und für sie ganz alltäglicher) Annahmen über Wärmestoff. Wärmestoff wird für eine

37 Vgl. auch Hacking (1983), Kap. 6.

38 Siehe Churchland (1985a), 286.

39 Siehe Churchland (1979), 16ff. Churchland führt die Gemeinschaft primär ein, um die systematische Fallibilität auch von Wahrnehmungsurteilen zu demonstrieren. Allerdings beruht sein Punkt wesentlich auf der Annahme, dass die Bedeutung der Urteile durch die Hintergrundtheorie bestimmt wird. Auf diese Annahme werde ich mich an dieser Stelle konzentrieren. 
feine, unsichtbare Flüssigkeit gehalten, die von Körpern in unterschiedlichem Maß aufgenommen werden kann. Zudem nehmen sie beispielsweise an, dass sich unterschiedlicher Wärmestoff-Druck in Körpern ausgleicht, wenn die Körper in Berührung kommen. Churchland verwendet einige Mühe darauf, die Alltagsannahmen der Wärmestoff-Freunde soweit anzureichern, dass sie eine Theorie ausmachen, die einen ziemlich großen Bereich von Temperaturphänomenen wie die Ausdehnung von Körpern bei Erwärmung oder einige Charakteristika des Temperaturausgleichs zwischen verschieden warmen Körpern ganz gut erklären kann.

Zudem kommen gemäß Churchlands Beschreibung Ausdrücke dieser Alltagstheorie wie ,hat einen hohen Wärmestoffdruck' und ,hat einen niedrigen Wärmestoffdruck' regelmäßig in den Beobachtungssätzen vor, die die Wärmestoff-Freunde unmittelbar in der Folge von Warm- bzw. Kalt-Empfindungen produzieren. Die Wärmestoff-Freunde urteilen demnach unmittelbar perzeptuell, dass Gegenstände einen hohen oder niedrigen Wärmestoffdruck haben. Die Ausdrücke dafür stehen für etwas (vermeintlich) direkt Wahrnehmbares.

Dieses Beispiel einer fiktiven Gemeinschaft scheint nahe zu legen, dass Ausdrücke, die wesentlich durch eine Rolle in einer weitreichenden explanatorischen Theorie charakterisiert sind, auch für perzeptuell Beobachtbares stehen können. Die Begründung der Netzwerk-Semantik ließe sich dann auf diese Ausdrücke übertragen. Damit wäre gezeigt, dass zumindest für einige Ausdrücke für perzeptuell Beobachtbares dieselbe Semantik wie für theoretische Ausdrücke gilt.

Gegen diese Argumentation habe ich allerdings einige Bedenken. Erstens ist unklar, ob das Beispiel tatsächlich menschlich möglich ist. Zunächst scheint zwar nichts dagegen zu sprechen. Es scheint möglich, dass die Wärmestofftheorie alltäglich akzeptiert wird. Warum sollten dann nicht perzeptuelle Beobachtungssätze in diesem Vokabular möglich sein? Mein Einwand richtet sich gerade gegen die Annahme, dass die Freunde des Wärmestoffs den unmittelbaren sinnlichen Eindruck haben können, dass da ein hoher Wärmestoffdruck herrscht. Die Schwierigkeit liegt dabei nicht darin, Drücke wahrzunehmen. Wir können Druck fühlen und so wahrnehmen. Aber ein Gefühl eines hohen Drucks ist von ganz anderer Art als eine Warm-Empfindung. Es scheint unmöglich, dass diese Warm-Empfindung Teil einer unmittelbaren Wahrnehmung eines hohen Drucks ist. Vielmehr kann sie nur Teil einer unmittelbaren Wahrnehmung einer hohen Temperatur sein. Dann kann es aber den Wärmestoff-Freunden aufgrund ihrer Temperatur-Empfindungen nicht unmittelbar sinnlich so vorkommen, als ob etwas einen hohen oder niedrigen Wärmestoffdruck hat. 
Aber selbst wenn die Ausdrücke der Wärmestofftheorie in den unmittelbaren Beobachtungssätzen vorkommen könnten, ist zweitens nicht klar, ob das regelmäßige Auftreten in solchen Sätzen ihre Semantik unverändert theoretisch belässt. Wenn sie als Beobachtungsausdrücke eingesetzt werden, könnten andere Faktoren ihre Bedeutung bestimmen als wenn sie für nicht perzeptuell Beobachtbares verwendet werden. Die lose Anbindung an Wahrnehmungen, die für theoretische Terme charakteristisch ist, fällt bei Beobachtungsausdrücken weg. Als Ausdrücke für perzeptuell Beobachtbares sind sie vielmehr durch eine Fähigkeit oder Neigung zu perzeptueller Klassifikation eng an bestimmte wahrnehmbare Phänomene geknüpft. Demgegenüber könnte ihre theoretische Rolle an semantischer Bedeutung verlieren. Theoretische Annahmen, die einstmals bedeutungsbestimmend waren, könnten zu bloßen Alltagsüberzeugungen über dasjenige werden, das wahrgenommen wird, ohne für die Bedeutung der Ausdrücke konstitutiv zu sein.

Die semantischen Unterschiede zwischen einem bloß theoretisch verwendeten Ausdruck für hohen Wärmestoffdruck und einem solchen Ausdruck für perzeptuell Beobachtbares könnten sich in unterschiedlichen Reaktionen auf die wissenschaftliche Entdeckung ausdrücken, dass die allgemeinen Annahmen über Wärmestoff falsch sind. Wir, die Wärmestoff weitgehend im Rahmen der Theorie verwenden, reagieren auf die Entdeckung, dass die Theorie empirisch widerlegt oder durch eine bessere Theorie ersetzt wird, mit der Feststellung, dass es Wärmestoff und damit hohe oder niedrige Drücke davon gar nicht gibt. Wir entdecken, dass der theoretische Ausdruck gar keine Referenz hat. Dies erscheint ganz vernünftig, da wir den Ausdruck dafür eingeführt haben, um eine einheitliche Erklärung von Temperaturphänomenen geben zu können. Wenn die Theorie damit scheitert, fallen die Gründe weg, an die Existenz des theoretisch Postulierten zu glauben.

Für die Wärmestoff-Freunde scheint bei derselben wissenschaftlichen Entdeckung aber eine ganz andere Reaktion angebracht. Sie würden wohl einfach zu Kenntnis nehmen, dass das Phänomen, das von ,hoher Wärmestoffdruck' beschrieben wird, gar kein Druckzustand einer Flüssigkeit ist, sondern tatsächlich einen hohen Grad der Ausschöpfung einer Kapazität, kinetische Energie aufzunehmen, bezeichnet. Zwar haben sich damit eine ganze Reihe theoretischer oder alltäglicher Annahmen als falsch herausgestellt. Aber es wäre für sie unsinnig anzunehmen, dass es gar nichts gab, das sie spürten und dessen verschiedene Konfigurationen sie mit TemperaturEmpfindungen wahrnahmen. Insbesondere überdauert ihre Fähigkeit, hohe von niedrigen Temperaturen perzeptuell zu unterscheiden, problemlos die Veränderung des theoretischen Verständnisses dessen, was da verlässlich unterschieden wird. Deshalb gibt es auch keinen Grund anzunehmen, dass sie 
mit ihren alten Beobachtungssätzen gar keine wirklich existierenden Phänomene unterschieden und beschrieben haben.

Es ist daher zweifelhaft, dass erstens die Freunde des Wärmestoffs überhaupt mit ihren Temperaturempfindungen unmittelbar den (vermeintlichen) Druck von Wärmestoff wahrnehmen können und dass zweitens, wenn sie dies tun sollten, die Semantik der betroffenen Ausdrücke unverändert durch die Wärmestofftheorie bestimmt wird. Aber selbst wenn man beides zugesteht, ist noch nicht klar, wie repräsentativ diese Situation ist. Ließe sich die Argumentation überhaupt auf die meisten Ausdrücken für perzeptuell Beobachtbares ausweiten? Hierfür müsste es für diese Ausdrücke zuallererst geeignete Theorien geben, die für die Bedeutungsfestlegung in Frage kämen. Churchland geht tatsächlich von der Existenz solcher Theorien aus. So behauptet er, dass auch unser gewöhnliches Temperaturvokabular wie ,warm oder ,kalt ${ }^{6}$ in ein Netzwerk alläglicher Annahmen eingebettet ist, die eine Theorie mit gewisser explanatorischer Kraft und einem recht breiten Anwendungsbereich bilden. ${ }^{40}$ Hierzu sollen solche Annahmen gehören wie die folgende:

(A) Von zwei Körpern desselben Gewichts ist derjenige wärmer, der einen dritten Körper am stärksten aufwärmen wird.

Feyerabend versucht in ähnlicher Weise allgemeine Annahmen auszumachen, die hinter der Bedeutung von alltäglichen Ausdrücken wie ,oben' und ,unten' oder Farbausdrücken stehen sollen. ${ }^{41} \mathrm{Zu}$ diesem Zweck führt er beispielsweise zwei populäre antike Probleme an, die sich daraus ergaben, dass man oben und unten als absolute Richtungen im Raum auffasste. Zum einen fragte man sich, wie es die Antipoden (die Bewohner der anderen Seite der Erde) schaffen, nicht von der Erde herunterzufallen, wenn man zugleich annimmt, dass die Erde kugelförmig ist. Zum anderen stellte man sich die Fragen, weshalb nicht die Erde selbst herunterfällt oder wodurch sie gestützt wird. Feyerabend behauptet, dass den Problemen u. a. die folgende kosmologische Annahme zugrunde liegt, die implizit im Alltag gemacht wurde:

(B) Der Raum ist anisotrop, d.h. er hat nicht in allen Richtungen dieselben physischen Eigenschaften.

40 Siehe Churchland (1979), 22ff. Churchland argumentiert zumindest an dieser Stelle aber nicht auf der Grundlage, dass es diese Theorien gibt, für die Netzwerk-Semantik. Vielmehr setzt er eine solche aufgrund einer Ausschluss-Argumentation wie der, die ich im folgenden Unterabschnitt vorstelle, schon voraus.

41 Siehe Feyerabend (1962), 85/86 zu ,oben'/,unten‘. Zu Farbausdrücken siehe Feyerabend (1960), 68/69. 
Allerdings ist hierbei erstens fragwürdig, ob diese Annahmen im Alltag tatsächlich gemacht wurden oder werden. Beide Annahmen scheinen mir sowohl zu allgemein als auch zu komplex zu sein, um sie zum Common Sense zu zählen. Annahme (A) bestimmt die Wärmemenge auf eine Weise, die zu differenziert ist. Annahme (B) enthält den Begriff der Anisotropie, über den der Alltagsverstand aber plausiblerweise nicht verfügt, auch nicht in impliziter Form.

Zweitens ist fragwürdig, dass diese Annahmen etwas zur Bedeutung der alltäglichen Ausdrücke, warm` bzw. ,oben' beitragen, wie sie in perzeptuellen Beobachtungsberichten vorkommen. Zunächst sind beide Annahmen falsch. Der Umfang der Erwärmung eines dritten Körpers hängt nicht nur an der Temperatur, sondern auch an der Wärmekapazität der ersten beiden Körper. Daher kann ein erster, weniger warmer Körper den dritten Körper stärker aufwärmen als ein zweiter Körper, wenn der erste eine größere Wärmekapazität hat. Unsere Beobachtungssätze scheinen aber von der Falschheit dieser Annahme völlig unberührt. Wenn unsere unmittelbaren Urteile vor der Entdeckung, dass (A) falsch ist, wahr waren, werden sie es auch danach sein, auch wenn dann (A) zurückgewiesen wurde. Dann hängen die Wahrheitsbedingungen dieser Sätze aber nicht an (A).

Ähnlich lässt sich mit Bezug auf (B) argumentieren. Die Wahrheit der Auskunft eines Atheners einem Touristen gegenüber, dass die Akropolis oberhalb der Stadt liege, ist völlig unberührt von der eventuellen Annahme von (B) in der Antike oder seiner späteren Zurückweisung. (B) trägt dann offenbar nichts zu den Wahrheitsbedingungen des alltäglichen Satzes über einen wahrnehmbaren Sachverhalt bei.

Dann wurden aber keine überzeugenden Gründe für die zweite Prämisse des Arguments von der Semantik theoretischer Ausdrücke gefunden. Zwar lassen sich Gründe für eine Netzwerk-Semantik für theoretische Ausdrücke für nicht perzeptuell Beobachtbares angeben. Es wurde aber nichts vorgebracht, das letztlich für die Annahme derselben Semantik bei Ausdrücken für perzeptuell Beobachtbares spricht. Allerdings haben Feyerabend und Churchland noch ein weiteres Argument, das dies begründen soll.

\section{c) Das Ausschluss-Argument}

Feyerabend und Churchland argumentieren für die Netzwerk-Semantik für Beobachtungsausdrücke auch, indem sie alternative semantische Theorien als inadäquat zurückweisen. ${ }^{42}$ Insbesondere zwei Arten alternativer Semantiken

42 Diese Argumentationsstrategie ist besonders deutlich in Feyerabend (1958) und Churchland (1979), $\mathbb{} 2$. 
werden dabei behandelt. Zum einen sind dies Ansätze, die die Bedeutung von Beobachtungssätzen an Empfindungen knüpfen, zum anderen solche, die äußere Umstände bzw. regel- oder gesetzmäßige Zusammenhänge zu diesen Umständen als konstitutiv für die Bedeutung von Beobachtungsausdrücken ansehen.

Empfindungen werden in der Regel für (relativ) theoriestabil gehalten. Wenn sie dazu beitragen, die Bedeutung von Beobachtungsausdrücken festzulegen oder wenn sie die Bedeutung einiger Ausdrücke vielleicht vollständig determinieren, würde dies die theoretische Varianz der Bedeutung der Ausdrücke einschränken oder ausschließen. Für eine solche Bedeutungsfestlegung kommen auf den ersten Blick Ausdrücke für sinnliche Eigenschaften wie , rot $^{6}$, süß $\beta^{6}$ oder ,warm' in Frage. Denn es gibt hier jeweils besondere Empfindungsarten, deren Vorkommnisse uns üblicherweise veranlassen, Sätze mit den Ausdrücken zu behaupten oder für wahr zu halten. Die Empfindungsarten werden daher in der Regel ebenfalls unter Verwendung des entsprechenden Ausdrucks charakterisiert. Rot-Empfindungen führen typischerweise dazu, „hier ist etwas Rotes“ für wahr zu halten, usw.

Feyerabend und Churchland leugnen eine solche Korrelation zwischen Vorkommnissen bestimmter Empfindungsarten und Sätzen nicht. Sie bestreiten aber, dass diese Korrelation die Bedeutung der Ausdrücke auch nur zum Teil festlegt. Churchland argumentiert hierfür, indem er die Möglichkeit von Wesen nachzuweisen versucht, bei denen Empfindungen mit anderen Ausdrücken korrelieren als bei uns. ${ }^{43}$ Die ,Infrarot-Seher ${ }^{6}$ haben, so geht die Fiktion, Augen, die nur für Infrarotstrahlung sensitiv sind. Die anschließende kognitive Verarbeitung führt bei ihnen zu Empfindungen der Art, die wir beim Sehen schwarzer oder weißer Gegenstände haben. Sie haben WeißEmpfindungen, wenn sie heißen Gegenständen begegnen, und SchwarzEmpfindungen, wenn etwas Kaltes in ihrem Blickfeld ist. Dennoch ist es möglich, so Churchland, dass sie auf die Empfindungen regelmäßig mit Sätzen reagieren, die „da ist etwas Heißes“ bzw. „da ist etwas Kaltes“ bedeuten. Um diese Möglichkeit zu plausibilisieren, gesteht Churchland den Infrarot-Sehern Temperatur-Überzeugungen ganz ähnlich den unseren zu. Sie glauben, dass Feuer heiß und Eis kalt ist, usw.

Wenn diese Fiktion möglich ist, zeigt dies, dass die Bedeutung von Ausdrücken, die regelmäßig in der Folge von Empfindungen verwendet werden, nicht schon dadurch notwendig in ihrer Bedeutung festgelegt sind. Bei denselben Empfindungen, auf die wir mit Behauptungen der Präsenz weißer Gegenstände reagieren, behaupten die Infrarotseher, dass da etwas Heißes ist.

43 Siehe Churchland (1979), $\$ 2$. 
Diese Behauptungen sind ihrem Inhalt nach grundsätzlich verschieden. Die Empfindungen scheinen demnach die möglichen Bedeutungen auch nicht wesentlich zu beschränken.

Aber auch die Unabhängigkeit der Bedeutung von „da ist etwas Heißes“ im Mund der Infrarot-Seher von ihren Weiß-Empfindungen lässt offen, ob nicht die Bedeutung mancher unserer Ausdrücke an Empfindungsqualitäten hängt. Jemand mag rote Gegenstände immer nur als mit Erdbeergeschmack erlebt haben. Wenn er daher einen roten Gegenstand auch nur sieht, urteilt er sofort: Da, etwas mit Erdbeergeschmack. Die Möglichkeit dieser Reaktion und die Unabhängigkeit der Bedeutung von der Empfindungsqualität zeigen aber nicht, dass die Bedeutung eines Satzes wie „da, etwas Rotes“ ebenfalls von der Empfindungsqualität unabhängig ist. Es ist demnach denkbar, dass das Temperaturvokabular der Infrarot-Seher seine Bedeutung auf andere Weise erhält als unser Farbvokabular.

Ob man eine semantisch konstitutive Rolle von Empfindungen für möglich hält, kann davon abhängen, wie man Empfindungsqualitäten auffasst. Man könnte von einer notwendigen Verknüpfung zwischen Empfindungsarten und intentionalen Gehalten ausgehen. ${ }^{44}$ Demnach ist es mit dem Haben einer Rot-Empfindung notwendig verknüpft, dass es mir sinnlich so vorkommt, als wäre da etwas Rotes. Natürlich brauche ich kein entsprechendes Urteil zu fällen. Ich komme vielleicht bloß zur Überzeugung, dass da etwas mit Erdbeergeschmack ist. Trotzdem ist es visuell so, als ob etwas Rotes vor mir wäre. Wenn Empfindungen - zumindest der Arten, die in sinnlichen Wahrnehmungen vorkommen - notwendig intentional sind, ist es nahe liegend anzunehmen, dass man mit einigen Ausdrücken den Inhalt von Empfindungen wiederzugeben versucht und deren Bedeutung daher zumindest teilweise durch die Empfindungen bestimmt wird. Dieser Position zufolge könnten die Infrarot-Seher zwar in der Folge von Weiß-Empfindungen behaupten, dass da etwas Heißes ist. Gleichzeitig haben ihre Empfindungen aber einen anderen intentionalen Gehalt und präsentieren weiße Gegenstände. Diesen Gehalt drücken wir aus, wenn wir in der Folge dieser Empfindungen behaupten, dass da etwas Weißes ist.

Diese Möglichkeit einer semantisch konstitutiven Rolle von Empfindungen ist aber versperrt, wenn man die Feyerabend-Churchland'sche Annahme teilt, dass Empfindungsarten lediglich durch intrinsische Qualitäten charakterisiert werden, die nicht notwendig mit intentionalen Eigenschaften verknüpft sind.

44 Von einer solchen notwendigen Verknüpfung gehen etwa Autoren aus, die phänomenale Qualitäten als identisch mit intentionalen Eigenschaften auffassen. Siehe etwa Harman (1990), Tye (1995), Dretske (1995). 
Denn dann scheint es zunächst plausibel anzunehmen, dass die Bedeutung von Beobachtungssätzen schon deshalb nicht durch eine Empfindungsqualität mitbestimmt wird, weil diese Sätze öffentliche Sachverhalte der Welt zum Inhalt haben, Empfindungsqualitäten jedoch Eigenschaften privater, mentaler Zustände sind. ${ }^{45}$ Allerdings gibt eine theoretische Option, um die Kluft zwischen semantisch konstitutivem mentalem Zustand und so festgelegtem öffentlichem Inhalt zu überbrücken. Einer klassischen Position zufolge sind Farbeigenschaften und andere sinnliche Eigenschaften relational. Die Eigenschaft, rot zu sein, besteht demnach beispielsweise in der Disposition, unter normalen Umständen bei normalen Beobachtern Empfindungen einer bestimmten intrinsischen Qualität - Rot-Empfindungen - hervorzurufen. Dieser Position zufolge behaupten wir mit „da ist etwas Rotes“ das Vorliegen einer dispositionalen Eigenschaft eines Gegenstands der Welt, also etwas Öffentliches, das dennoch wesentlich die intrinsische Qualität mentaler Zustände einschließt. Demzufolge konstatieren die Infrarot-Seher vermutlich mit ihrem „da ist etwas Heißes“ das Vorliegen einer anderen Eigenschaft, als wir es mit einem entsprechenden Satz tun. Denn ihre Äußerung bezieht sich vermutlich auf die Disposition, bei gewöhnlichen Infrarot-Sehern WeißEmpfindungen hervorzurufen. Dagegen nimmt unsere Behauptung Bezug auf die Disposition, bei normalen Menschen Heiß-Empfindungen hervorzurufen. ${ }^{46}$

Dies zeigt, dass die Feyerabend-Churchland'sche Zurückweisung der semantischen Konstitutivität von Empfindungen an speziellen Annahmen zur Natur von Empfindungen und sinnlichen Eigenschaften hängt. Empfindungen dürfen demnach als solche nicht intentional sein, und sinnliche Eigenschaften sollen keine Relationen zu Empfindungsarten sein. Aber auch wenn man diese Annahmen zurückweisen könnte, käme nur eine recht beschränkte Menge von Prädikaten für eine Bedeutungsfestlegung durch Empfindungen in Frage. Es sind dies Prädikate, die sehr direkt einzelnen perzeptuellen Empfindungen (Farb-, Geräusch-, Geschmacks- etc. Empfindungen) zugeordnet werden können und insofern sinnliche Eigenschaften bezeichnen. Viele andere Prädikate, die alltägliche oder wissenschaftliche beobachtbare Eigenschaften, Stoffe oder Arten bezeichnen - von ,stabil‘ oder ,durchsichtig' über ,Milch' und ,Schlamm` zu ,Kirschbaum' und ,Hund' - kommen für eine solche semantische Position zunächst sowieso nicht in Frage.

45 Vgl. Feyerabend (1960), Abschn. 3.

46 Für eine solche Antwort auf Churchlands Beispiel der Infrarot-Seher siehe Tagliaferro (1991). Vgl. Churchland (1979), 12/13 für Überlegungen hierzu. 
Das Churchland'sche Beispiel der Infrarot-Seher scheint aber eine andere semantische Position nahe zu legen, deren Anwendungsbereich nicht so beschränkt zu sein braucht und derzufolge die Bedeutung von Beobachtungsausdrücken ebenfalls weitgehend theorieunabhängig wäre. Die Sätze der Infrarotseher mit der Bedeutung, dass da etwas Heißes ist, werden regelmäßig im Laufe von Sinneserfahrungen produziert, die von heißen Gegenständen verursacht werden. Dies ist bei normalen Menschen nicht anders. Zwar führen heiße Gegenstände bei gewöhnlichen Menschen zu anderen Empfindungen als bei Infrarotsehern, die jeweils folgenden Beobachtungssätze haben aber dieselbe Bedeutung. Dies legt die Idee nahe, dass - grob gesagt - die regel- oder gesetzmäßigen Korrelationen von Vorkommnissen heißer Gegenstände und Vorkommnissen des Satzes „da ist etwas Heißes“ die Bedeutung des Satzes festlegen.

Es gibt eine ganze Familie von semantischen Positionen, die davon ausgehen, dass die Bedeutung eines Beobachtungsausdrucks oder -satzes $A$ durch die Eigenschaft $F$ festgelegt wird, mit deren Instanzen die Vorkommnisse des Ausdrucks nomologisch verbunden sind. So hat Dretske eine informationale Semantik vorgeschlagen, wonach die Bedeutung einiger Ausdrücke oder mentaler Zustände durch die Information mit festgelegt wird, die sie tragen. Dabei trägt der Ausdruck $A$ die Information, dass ein $F$ vorliegt, wenn es nomologisch wahr ist, dass wenn $A$ vorkommt, auch ein $F$ vorliegt. ${ }^{47}$ Fodor hat die Position vertreten, dass der Ausdruck $A$ die Eigenschaft $F$ genau dann zum Inhalt hat, wenn gilt:

1. Instanzen von $F$ verursachen unter optimalen Bedingungen in Beobachtern Vorkommnisse von $A$.

2. Falls Dinge, die nicht $F$ sind, auch $A$ s verursachen, hängt diese Verursachung asymmetrisch von der Verursachungsrelation zwischen $F$ und $A$ ab. ${ }^{48}$

Dabei ist eine zweite Verursachungsrelation von einer ersten asymmetrisch abhängig genau dann, wenn die erste auch bestehen würde, wenn die zweite nicht bestünde, die zweite aber ohne die erste nicht bestehen würde. Wenn tatsächlich neben $F$ s auch nicht- $F$ s $A$ s verursachen, dürfen demnach in den nächsten möglichen Welten, in denen Fs keine As verursachen, auch die fraglichen nicht-Fs keine $A$ s verursachen. Gleichzeitig müssen in den nächsten möglichen Welten, in denen die nicht-Fs keine As verursachen, die As dennoch von $F$ s verursacht werden.

\footnotetext{
47 Siehe Dretske (1981). Dretske zufolge bedeutet ein Ausdruck nicht alle Information, die er trägt, sondern nur eine Teilmenge davon. Vgl. Beckermann (2001), 334ff.

48 Siehe Fodor (1987), Kap. 4, und Fodor (1990), Kap. 4; vgl. Beckermann (2001), 349ff.
} 
Beide Positionen sind in vielen Hinsichten genauer spezifizierbar. Für den gegenwärtigen Kontext ist aber zunächst bemerkenswert, dass die Positionen zur Folge haben, dass die Bedeutung von Beobachtungsausdrücken weitgehend oder vollständig von Theorien unabhängig ist. Denn die nomologischen Relationen zwischen $F$ und $A$ bestehen unabhängig von Theorien.

An dieser Stelle ist keine vollständige Würdigung dieser Positionen möglich. Feyerabend diskutiert kausale Bedeutungstheorien zwar recht ausführlich unter der Bezeichnung, pragmatisches Sinnprinzip'.49 Allerdings legt er sehr schlichte Versionen der Bedeutungstheorie zugrunde, so dass eine sinnvolle Übertragung der Diskussion auf die ausgefeilten Positionen Dretskes und Fodors nicht sinnvoll ist. Churchland geht an einigen Stellen kurz auf solche Theorien ein. Insbesondere formuliert Churchland einen Einwand, den ich hier diskutieren möchte. Churchland bezieht sich ursprünglich auf eine frühe Version von Dretskes Vorschlag und wendet ein, dass der Vorschlag es unmöglich zu machen scheint, dass sich Beobachter systematisch irren. Als Beispiele führt Churchland Beobachtungssätze wie ,die Götter schreien' und ,da sind Fenster in der Kristallsphäre ${ }^{6}$ an. ${ }^{50}$ Man kann hier annehmen, dass diese Sätze in einer Sprachgemeinschaft aufgrund einer nomologischen Relation regelmäßig beim Hören von Donnern bzw. Sehen von Sternen geäußert werden. Dennoch müssen die Sätze nicht bedeuten, dass es donnert oder dass da Sterne stehen. Vielmehr können sie einen Inhalt haben, der immer und systematisch falsch ist. Churchland geht aber davon aus, dass Positionen der besprochenen Art mit ständigem und systematischem Irrtum unvereinbar sind. Diese Positionen scheinen, indem sie die Bedeutung von Sätzen oder Ausdrücken an eine nomologische Korrelation mit äußeren Umständen binden, es semantisch unmöglich zu machen, dass ein Satz immer falsch ist. Wenn man es aber wie Churchland von allen unseren Beobachtungssätzen für möglich hält, dass sie systematisch falsch sind, kann man schließen, dass diese semantischen Positionen für Beobachtungssätze ungeeignet sind.

Wie gesagt, formuliert Churchland seinen Einwand gegen eine frühe Fassung der Position Dretskes. Im Weiteren hat Dretske seine Position weiter entwickelt und dabei einen Schwerpunkt auf die Möglichkeit von Irrtümern gelegt. ${ }^{51}$ Aber auch Fodor hat sich des Problems angenommen. Ich möchte jetzt untersuchen, wie weit der Churchland'sche Einwand gegen Fodors Position trägt.

\footnotetext{
49 Siehe insbes. Feyerabend (1958).

50 Siehe Churchland/ Churchland (1983), 67.

51 Siehe Dretske (1986).
} 
Hierbei sind zunächst zwei Bemerkungen zum intendierten Anwendungsbereich von Fodors Theorie angebracht. Erstens formuliert Fodor seine Position nicht nur oder nicht vorzugsweise für sinnliche Eigenschaften wie Röte oder Wärme, sondern für Eigenschaften, die alltägliche und wissenschaftliche Klassen und Stoffe charakterisieren, wie anhand seiner Beispiele klar wird (Pferd und auch Proton). ${ }^{52}$ Fodors Position ist daher ein gutes Beispiel für eine semantische Theorie, die nicht auf Ausdrücke für sinnliche Eigenschaften beschränkt ist.

Zweitens formuliert Fodor seine Position für syntaktisch primitive Ausdrücke, die in elementaren Sätzen wie ,das ist ein Pferd' vorkommen. Die Churchland'schen Beispiele sind schon deswegen als Fälle für Fodors Position ungeeignet, weil es recht komplexe Sätze sind, in denen mehrere Begriffe vorkommen. Vielleicht wären statt, die Götter schreien' und ,da sind Fenster in der Kristallsphäre` solche Äußerungen wie ,da, Göttergeschrei!‘ und ,da, Himmelsfenster!' besser für die Diskussion geeignet. Aber die Prädikate sind hier zumindest im Deutschen immer noch syntaktisch komplex. Besser scheint es, andere Beispiele zu wählen, die nicht im Verdacht syntaktischer Komplexität stehen. Man braucht hierzu den Bereich des Okkulten nicht zu verlassen, vielmehr bieten sich Ausdrücke wie ,Hexe', ,Meeresungeheuer' oder ,Ufo' an (oder ,Drache', ,Gespenst ${ }^{6}$,Irrlicht', ,Nessie' etc.). Es fällt wohl nicht schwer, sich in eine Zeit zurückzuversetzen, in der diese Ausdrücke - mit im Großen und Ganzen unveränderter Bedeutung - regelmäßig in der Folge bestimmter Erscheinungen oder für Menschen bestimmten Aussehens verwendet wurden. Beispielsweise wurde ,Hexe ${ }^{6}$ regelmäßig auf rothaarige Frauen, die einen bestimmten wirren Blick hatten, angewendet. (Ich vereinfache die komplexen Anwendungsbedingungen stark. ${ }^{53}$ ) Alle diese Äußerungen waren falsch: Es gab keine Hexen. Aber innerhalb der Gemeinschaft verursachten Frauen des beschriebenen Aussehens systematisch solche Äußerungen. Ihr Ausdruck ,Hexe` ist nomologisch mit den Frauen verknüpft.

52 Fodor (1987), Kap. 4.

53 Für die gewöhnliche Vorstellung von Hexen siehe den Artikel ,Hexe' in BächtoldStäubli (Hg.) (1931), Bd. III, insbes. 1896/1897: „Aussehen: Eine Hexe ist meist eine alte hässliche Frau ... mit roten, und entzündeten, tiefliegenden und rotgeschwollenen, von roten, fleischigen Ringen umgebenen, triefenden oder leuchtenden Augen und mit zusammengewachsenen Augenbrauen. Alle Hexen blinzeln. Sie sind schmutzig, haben zerzaustes Haar, einen Bart, Schnurrbart, ein spitzes Kinn und sprechen wie ein Mann. Sie sind mager, bleich, erdfarben, hinken und haben einen Buckel, eine krumme Nase, die bis ans Kinn reicht..." 
Die Bedeutung von ,Hexe' und ähnlich gelagerten Beispielen stellt Fodors Position vor zwei Schwierigkeiten. ${ }^{54}$ Erstens gibt es keine Hexen. Also gibt es keine Hexen, die tatsächlich ,Hexe-Vorkommnisse verursachen. Fodors Vorschlag kann aber im Prinzip mit diesem Problem zurechtkommen. ${ }^{55}$ Denn Fodor betrachtet eine nomologische Korrelation zwischen Eigenschaften als semantisch bestimmend. Solche Korrelationen können aber auch zwischen Eigenschaften bestehen, die tatsächlich nicht instanziiert sind. Wichtig ist daher, dass es, wenn es Hexen gäbe, diese Hexen „Hexe'-Vorkommnisse verursachen würden. Genauer gesagt muss Fodor davon ausgehen, dass gilt:

(1) In den nächsten Welten, in denen es Hexen gibt, verursachen diese ,Hexe'Vorkommnisse.

Die zweite Schwierigkeit ergibt sich daraus, dass die tatsächlichen Vorkommnisse von ,Hexe von rothaarigen, wirr blickenden Nicht-Hexen verursacht werden. Warum bedeutet ,Hexe' dann nicht einfach rothaarige, wirr blickende Nicht-Hexe, sondern Hexe? Doch auch für dieses Problem hat Fodor im Prinzip eine Lösung, die in der obigen Präsentation von Fodors Position schon enthalten ist. ,Hexe' bedeutet dennoch Hexe und nicht rothaarige Frau mit wirrem Blick, wenn die Verursachungsrelation zwischen rothaarigen, wirr blickenden Frauen und „Hexe' asymmetrisch von der Verursachungsrelation zwischen Hexen und ,Hexe' abhängt. Dies schließt die Behauptung ein (siehe die obige Vorstellung der Position):

(2) In den nächsten möglichen Welten, in denen es Hexen gibt, die keine ,Hexe'Vorkommnisse verursachen, verursachen auch rothaarige, wirr blickende Frauen keine ,Hexe'-Vorkommnisse.

Beide Lösungsansätze sind aber im Fall von ,Hexe (und allen Fällen dieser Art) unbrauchbar. Zunächst ist es unklar und in der Tat unwahrscheinlich, dass Hexen, wenn es sie gäbe, überhaupt ,Hexe'-Vorkommnisse verursachen würde. Denn die Verursachung tatsächlicher ,Hexe'-Vorkommnisse macht

54 Putnam kritisiert Fodors Position ebenfalls anhand einer Diskussion des Beispiels ,Hexe'. Allerdings beruht seine Kritik nicht auf dem Churchland'schen Einwand, dass Beobachtungssätze systematisch falsch sein können. Vielmehr untersucht Putnam, ob der Begriff der Hexe überhaupt kohärent ist. Er bezweifelt dies, und kommt zum Schluss, dass das subjunktive Konditional „wenn es Hexen gäbe, würden sie ,Hexe“Vorkommnisse verursachen“ daher nicht wahr sein kann. Fodor braucht die Wahrheit dieses Konditionals aber für die Erfüllung der ersten Bedingung der oben dargestellten Position. Siehe Putnam (1992), 43ff. Ich gehe im Folgenden in der Diskussion des Beispiels aber davon aus, dass Hexen metaphysisch und auch nomologisch möglich sind.

55 Vgl. Fodors Diskussion von ,Einhorn‘, Fodor (1990), 100/101. 
sich am Aussehen vermeintlicher Hexen fest: Die Vorkommnisse werden verursacht von Frauen, die rothaarig sind und wirr blicken. Eine Hexe wird aber letztlich nicht durch ihr Aussehen charakterisiert, sondern dadurch, ob sie beispielsweise über außergewöhnliche Kräfte oder Fähigkeiten verfügt, mit dem Teufel im Bunde steht oder Ähnliches. Es gibt keine notwendige Verbindung zwischen diesen, inneren' Eigenschaften und ihrem Aussehen. Die populären Vorstellungen nicht nur darüber, dass es Hexen gibt, sondern wohl auch darüber, wie sie aussehen würden, wenn es sie gäbe, sind völlig irrig. Es ist daher extrem unwahrscheinlich, dass Hexen in der möglichen Welt, die der unseren am nächsten ist und in der es welche gibt, überwiegend rothaarige, wirr blickende Frauen sind. Dann ist es aber extrem unwahrscheinlich, dass sie ,Hexe'-Vorkommnisse verursachen würden.

So unwahrscheinlich es ist, dass Hexen ,Hexe'-Vorkommnisse verursachen würden, so wahrscheinlich ist es, dass rothaarige, wirr blickende Frauen dies tun würden, auch wenn die Hexen dies nicht tun würden. In der nächsten möglichen Welt mit Hexen, die keine ,Hexe'-Vorkommnisse verursachen, sind Hexen vielleicht überwiegend blond und blicken gutmütig. Es wäre anzunehmen, dass trotzdem rothaarige, wirr blickende Nicht-Hexen ,Hexe'Vorkommnisse verursachen. Jedenfalls wäre eine solche mögliche Welt, in der diese Kausalrelation besteht, unter sonst gleichen Umständen der unseren Welt ähnlicher als eine, in der sie nicht besteht. Doch dann ist auch die zweite Annahme, die von Fodor in Anspruch genommen werden muss, falsch. Es ist insgesamt unglaubwürdig, dass eine Verursachungsrelation zwischen Hexen und ,Hexe' besteht. Diese besteht zwischen rothaarigen, wirr blickenden Frauen und ,Hexe. Wenn aber die erste Relation besteht, dann ist die zweite nicht davon asymmetrisch abhängig. Es ist vielmehr umgekehrt: Wenn die erste Relation besteht, dann deswegen, weil Hexen (zufällig) rothaarig und wirr blickend aussehen und solche Frauen ,Hexe'-Vorkommnisse verursachen.

Das Argument der letzten beiden Abschnitte gegen eine Fodor'sche Semantik für ,Hexe` lässt sich verallgemeinern. Betrachten wir eine Eigenschaft $F$ von möglichen Dingen, die sich nicht hauptsächlich in Oberflächeneigenschaften der Dinge ausdrückt, sondern eine, innere' Eigenschaft ist. (Dazu zählen die Eigenschaften, eine Hexe zu sein genauso wie die, ein Hund zu sein oder ein Proton zu sein.) Nehmen wir an, die Eigenschaft ist nicht tatsächlich instanziiert. Dennoch kann es einen primitiven Ausdruck $A$ geben, der diese Eigenschaft bezeichnet. Dann ist es aber möglich, dass eine Klasse tatsächlicher Dinge mit der Oberflächeneigenschaft $G$ regelmäßig diesen Ausdruck verursacht. Es ist möglich, dass wir uns ständig und systematisch darin irren, dass etwas $F$ ist. Die Bedeutung dieses Ausdrucks wird aber dann 
nicht in der Weise festgelegt, die Fodor vorschlägt. Denn die Kausalrelation besteht zuallererst zwischen den tatsächlichen Dingen, die $G$ sind, und $A$. Es ist erstens unklar, ob überhaupt eine Kausalrelation zwischen $F$ und $A$ besteht. Und falls eine solche Kausalrelation besteht, ist es zweitens unglaubwürdig, dass die Relation zwischen $G$ und $A$ asymmetrisch davon abhängt. Vielmehr ist zu vermuten, dass die Abhängigkeit in umgekehrter Richtung besteht. ${ }^{56}$

Wenn es aber von allen unseren Beobachtungsausdrücken, die nicht rein sinnliche oder Oberflächeneigenschaften bezeichnen, möglich ist, dass sie nicht tatsächlich instanziiert sind und wir uns in ihrer Anwendung ständig und systematisch irren, dann kann die Bedeutung aller dieser Ausdrücke nicht auf Fodor'sche Weise festgelegt sein. Denn Fodors Weise der Bedeutungsfestlegung ist mit einer solchen Situation unvereinbar. Die Situation müsste demnach semantisch unmöglich sein.

Insgesamt zeigt sich, dass semantische Positionen, die nomologische Korrelationen zwischen Vorkommnissen von Ausdrücken und Eigenschaften oder Tatsachen als semantisch konstitutiv auszeichnen, durch die Möglichkeit systematisch falsch angewendeter Ausdrücke, die nichts tatsächlich Bestehendes bezeichnen, vor hohe Hürden gestellt werden. Positionen, die demgegenüber auf Empfindungen bauen, können dagegen auch für den Fall, dass Empfindungen oder sinnliche Eigenschaften eine geeignete Natur haben, allenfalls für ein sehr beschränktes Vokabular semantisch bestimmend sein. Feyerabend und Churchland schließen aus den Schwächen dieser alternativen Theorien, dass die Netzwerk-Semantik auch für Ausdrücke für perzeptuell Beobachtbares die beste theoretische Option darstellt. Dieser Schluss ist aber nicht zwingend. Erstens könnte die Netzwerk-Semantik selbst bei Beobachtungsausdrücken mit Problemen konfrontiert sein; zweitens könnte es eine weitere Alternative geben, die erfolgreich ist. Diese Möglichkeiten, die Geltung des Ausschluss-Arguments zu untergraben, werde ich aber erst in Kapitel 7 ausloten.

56 Vgl. Fodor (1987), 121/122: Fodor erwägt hier, dass die Kausalrelationen zwischen Gegenständen, die nicht durch ihre Oberflächeneigenschaften wesentlich charakterisiert sind, und Ausdrücken für diese Gegenstände vermittels der Oberflächeneigenschaften und Ausdrücken für diese Eigenschaften bestehen. Dies ermöglicht aber gerade den diskutierten Einwand. 


\section{Die syntaktische Theorie der Beobachtung und der Pessimismus}

\section{a) Zusammenfassung der Position}

Wenn man die Argumentation, wie sie bisher präsentiert und vorläufig akzeptiert wurde, zusammenfasst, ergibt sich die folgende Position zur Theoriebeladenheit der Beobachtung. Erstens werden Beobachtungen im Allgemeinen nach der Funktionsweise von Messgeräten modelliert: Sie sind Kausalprozesse, die zu Beobachtungssätzen führen. Die Kausalrelation macht sich dabei an der Syntax der Beobachtungssätze fest. Beobachtungen haben zudem keine weiteren Ergebnisse, die epistemisch von Bedeutung sind.

Zweitens wird angenommen, dass die Bedeutung der Beobachtungssätze durch die inferenziellen Relationen festgelegt wird, in denen sie (bzw. die darin vorkommenden Ausdrücke) gemäß den geltenden Theorien stehen. Die Argumentation für diese Position ist komplex und mit vielen Fragezeichen zu versehen. Insgesamt aber wurde zunächst dafür argumentiert, dass eine solche Netzwerk-Semantik für Ausdrücke, die wesentlich in explanatorischen Theorien vorkommen und nichts perzeptuell Beobachtbares bezeichnen, plausibel ist. Dann wurde behauptet, dass Ausdrücke für perzeptuell Beobachtbares semantisch genauso funktionieren. Dafür konnten bei Feyerabend und Churchland aber keine überzeugenden Gründe gefunden werden. Die Argumentation lässt sich aber verstärken, indem man einige alternative semantische Positionen zu Wahrnehmungsausdrücken zurückweist. Zum einen beruht die Churchland'sche Zurückweisung auf der Ablehnung bestimmter Positionen zur Natur von Empfindungen und sinnlichen Eigenschaften. Zum anderen habe ich einige Ideen Churchlands zu einem Einwand gegen Fodors kausale semantische Position ausgebaut. So entstand insgesamt eine Argumentation, die die Netzwerk-Semantik für Beobachtungsausdrücke jedenfalls nicht von vornherein unplausibel erscheinen lässt. Allerdings ist die Begründung anfällig insbesondere gegenüber der Präsentation einer möglichen weiteren, alternativen semantischen Position für Beobachtungsausdrücke.

Damit ergibt sich folgende Vorstellung von Beobachtungen: Beobachtungen führen zu Beobachtungssätzen als wissenschaftlich verwertbaren Ergebnissen. Die Bedeutung der Beobachtungssätze ist aber völlig unabhängig von ihrer kausalen Genese. Vielmehr wird die Bedeutung vollständig durch das theoretische Netzwerk festgelegt. Zu diesem Netzwerk gehören insbesondere die Theorien, welche die Beobachtungen erklären sollen. Man kann daher die Tatsache, welche Beobachtungssätze in einer Beobachtung produziert werden, von der Tatsache trennen, welche Bedeutung diese Sätze durch die Einbettung in Theorien erhalten. Insbesondere können die Kausalprozesse, 
die zu Beobachtungssätzen führen, gegenüber theoretischem Wandel stabil sein. Alternative Theorien zu einem Gegenstandsbereich können mit derselben Menge von Beobachtungssätzen konfrontiert werden. Allerdings erhalten die Beobachtungssätze durch verschiedene Theorien unterschiedliche Bedeutungen. Die alternativen Theorien stehen also nur insofern denselben Sätzen gegenüber, als diese uninterpretiert sind, d.h. bloß syntaktisch spezifiziert sind. Die Beobachtungsprozesse legen daher nur die Syntax der Beobachtungssätze und die Zeitpunkte, zu denen sie produziert werden, fest. Wegen der Weise, in der äußere Umstände die Produktion von Beobachtungssätzen bestimmen, nennt Feyerabend diese Auffassung von Beobachtungen die „,pragmatische Theorie“ ${ }^{\circ 57} \mathrm{Da}$ diese äußeren Ursachen aber, wie gesehen, bloß die Syntax der Sätze festlegen und ihr Inhalt damit durch empirische Faktoren völlig unbestimmt bleibt, scheint die Bezeichnung als syntaktische Theorie angebrachter. Im Folgenden will ich Beobachtungen, die entsprechend der syntaktischen Theorie beschaffen sind, als Feyerabend-Churchland-Beobachtungen bezeichnen.

Stützen Feyerabend-Churchland-Beobachtungen und ihre Theorieabhängigkeit einen epistemologischen Pessimismus? Dann könnte man gegenüber den Erkenntnisaussichten der Wissenschaften nicht mehr optimistisch sein. Feyerabend und Churchland selbst nehmen hier verschiedene Positionen ein. Zwar hat Feyerabend seine philosophische Karriere als Realist begonnen. Allerdings schließt seine Variante des Realismus nicht explizit einen epistemischen Optimismus ein. Vielmehr ist sein Realismus durch die Behauptung charakterisiert, dass wissenschaftliche Theorien wörtlich verstanden werden sollten, also als Annahmen über in der Regel unbeobachtbare Entitäten, und nicht etwa instrumentalistisch. ${ }^{58}$ Später hat sich Feyerabend zum Anarchisten gewandelt und äußert sich explizit pessimistisch über die Erkenntnisaussichten der Wissenschaften. ${ }^{59}$

Churchland gibt sich dagegen oft als optimistischer wissenschaftlicher Realist aus. ${ }^{60}$ Besonders interessant ist hierbei, dass er die Theoriebeladenheit von Beobachtungen nicht vorwiegend als Bedrohung der wissenschaftlichen Objektivität, sondern als epistemische Chance auffasst. Er glaubt, dass die gegenwärtigen Beobachtungen und Wahrnehmungen häufig irreführend und in ihrer Reichweite unnötig eingeschränkt sind. Indem wir unsere Beobachtungen von den besten verfügbaren Theorien beeinflussen lassen, soll ihre

\footnotetext{
57 Siehe Feyerabend (1962), 36.

58 Siehe etwa Feyerabend (1960a). Vgl. Preston (1997), 61ff.

59 Siehe Feyerabend (1975), z.B. 34 u. 49/50.

60 Siehe insbes. Churchland (1979).
} 
Verlässlichkeit erhöht und insbesondere der Bereich dessen, was wir beobachten können, erweitert werden.

Diese Idee erscheint zunächst attraktiv. Die epistemische Qualität theoriebeladener Beobachtungen hängt auch von der Qualität der Theorien ab, die sie beladen. Wenn wir Beobachtungen durch gute Theorien beladen, können wir die Beobachtungen so vielleicht verbessern. Allerdings ist hier auch Vorsicht geboten. Verhindert ein Übermaß an Theoriebeladenheit nicht schon, dass wir zuallererst zu guten Theorien kommen? Ich werde gleich zur Frage kommen, ob das $\mathrm{Maß}$ an Theorieabhängigkeit, das Feyerabend und Churchland vertreten, selbst dem möglichen epistemischen Ertrag, den Theoriebeladenheit erbringen kann, die Grundlage nimmt. Zunächst aber werde ich Bedenken gegenüber dem Optimismus ausarbeiten, die Churchland selbst artikuliert hat.

\section{b) Das Heuhaufen-Argument}

Wie gesehen, legt der vorgestellten Position zufolge der Beobachtungsprozess die Beobachtungssätze nur hinsichtlich ihrer Syntax fest. Wissenschaftler sind dann vor die Aufgabe gestellt, eine Theorie zu formulieren, die diese Beobachtungen sowohl erklärt als auch deutet. Ein Optimist muss davon ausgehen, dass die Wissenschaft hierbei unter geeigneten Umständen mit hoher Wahrscheinlichkeit zu einer Theorie kommen wird, die überwiegend wahr ist. Churchland hat aber das Bedenken geäußert, dass diese Suche nach einer wahren Theorie der Aufgabe gleicht, ,eine Nadel in einem gewaltigen Heuhaufen" zu finden. Es gibt Churchland zufolge eine schier unermessliche Anzahl von Theorien (bzw. begrifflicher Schemata), die denkbar sind. Es sei daher extrem unwahrscheinlich, dass wir jemals auf die richtige, d.h. eine überwiegend wahre Theorie stoßen werden. Deshalb müsse man gegenüber den Erkenntnisaussichten der Wissenschaften letztlich pessimistisch sein. ${ }^{61}$

Churchland formuliert dieses Bedenken vor dem Hintergrund seiner konnektionistischen Vorstellung wissenschaftlicher Theorien. Zudem diskutiert er bloß die Frage, wie wahrscheinlich es ist, eine wahre Theorie über alles (,a final, true theory of the cosmos") zu finden. Sein Heuhaufen-Argument ist aber unabhängig von diesen Voraussetzungen. Es ist auch für Theorien gültig, die nur einen eingeschränkteren Anwendungsbereich haben, und es lässt sich auf der Grundlage der allgemeinen Position zu Beobachtungen und ihrer Semantik formulieren, die Gegenstand dieses Kapitels ist.

Das Heuhaufen-Argument beruht zum einen auf einer Abschätzung der Zahl denkbarer Theorien, zum anderen auf der Charakterisierung der epis-

61 Siehe Churchland (1996), insbes. 42. 
temischen Rolle, die Beobachtungen im Forschungsprozess spielen. Churchland errechnet die Zahl denkbarer Theorien auf der Grundlage seiner konnektionistischen Theorie des Geistes. Jede Verteilung von Gewichtungen der Verbindungen, die zu einer spezifischen Einteilung des Raums möglicher Aktivitäten führt, hat zur Folge, dass das Netzwerk die Repräsentationen auf besondere Weise verarbeitet. Aus der Sicht der konnektionistischen Netzwerk-Semantik ist es daher nicht unplausibel, die spezifischen Verteilungen der Gewichte als Repräsentationen besonderer Theorien bzw. begrifflicher Schemata aufzufassen. Churchland kommt mit der Annahme, dass die geschätzten $10^{11}$ Neuronen und $10^{14}$ Synapsen des menschlichen Gehirns ein konnektionistisches Netzwerk sind, von denen jede Synapse zehn mögliche Gewichtungen hat, zur Abschätzung, dass es die astronomische Anzahl von 10 hoch $10^{14}$ oder $10^{100.000 .000 .000 .000}$ möglicher Verteilungen von Gewichtungen oder denkbaren Theorien gibt. ${ }^{62}$

Aber auch wenn man die Schätzung etwas konservativer auslegt und nicht an die Annahmen des Churchland'schen Konnektionismus bindet, kommt man ausgehend von einer gewöhnlichen Netzwerk-Semantik auf eine große Anzahl denkbarer Theorien. Angenommen, wir haben eine mittelgroße Theorie mit 30 Begriffen. Jeder dieser Begriffe stehe in zehn inferenziellen Relationen mit anderen Begriffen. Hierbei seien drei verschiedene Relationen möglich, etwa Koinstanziierung (,A immer genau dann, wenn B ), kausale Folge (,wenn A, dann folgt kurz darauf B) und Oberbegriff-Unterbegriff (,alle A sind B). Insgesamt bestehen also 150 inferenzielle Relationen: Jeder der 30 Begriffe steht in 10 Relationen, in jeder Relation stehen zwei Begriffe. Die Anzahl der möglichen Relationen beträgt 1305, denn jeder der 30 Begriffe kann mit 29 anderen Begriffen in drei Relationen stehen, die je zwei Begriffe umfassen, und (30.29·3) $/ 2=1305$. Es kann also eine Auswahl von 150 Relationen aus 1305 bestehen. Demnach sind 1305 über $150=$ 1305!/(1155!·150!) Kombinationen möglich. Eine Abschätzung der Größe ergibt, dass dies mehr als $10^{187}$ mögliche Kombinationen sind. ${ }^{63}$

Es ist also auf der Grundlage einer Netzwerk-Semantik anzunehmen, dass es eine unüberschaubar immense Anzahl denkbarer Theorien auch für beschränkte Anwendungsbereiche gibt. Die weitaus meisten dieser Theorien werden in großen Teilen falsch sein. Es ist von einer beliebig herausgegriffenen Theorie sehr unwahrscheinlich, dass sie überwiegend wahr ist. Um wissenschaftlich erfolgreich sein zu können, muss man daher dazu kommen,

62 Siehe Churchland (1996), $10 \mathrm{ff}$.

${ }_{63}$ Es ist: $\quad 1$. 1305! $>1155 ! \cdot 1156^{150}>1155 ! \cdot 1000^{150}=1155 ! \cdot 10^{450}$.

2. $150 ! \approx 5,7 \cdot 10^{262}<10^{263}$.

Daher ist $1305 ! /(1155 ! \cdot 150 !)>\left(1155 ! \cdot 10^{450}\right) /\left(1155 ! \cdot 10^{263}\right)=10^{187}$. 
Theorien in Betracht zu ziehen, die eine überdurchschnittliche Wahrscheinlichkeit darauf haben, überwiegend wahr zu sein. Wenn man nur durchschnittlich wahrscheinliche Theorien testet, wird man auch nach sehr langer Zeit nicht mit hoher Wahrscheinlichkeit auf eine überwiegend wahre Theorie gestoßen sein.

Von Feyerabend-Churchland-Beobachtungen ist aber keine Hilfe darin zu erwarten, die Wahrscheinlichkeit der Wahrheit von in Betracht gezogenen Theorien zu erhöhen. Denn solche Beobachtungen führen, wie gesehen, zu Sätzen, die qua Beobachtungssätze inhaltlich völlig unbestimmt sind. Man kann sich die Sätze als von der Form, hier ist jetzt ein $F^{k}$ oder auch ,zum Zeitpunkt $t$ befindet sich am Ort $\left(x_{2}, y, z\right)$ ein $F^{k}$ vorstellen. Wenn man diese Sätze nicht durch eine schon vorgegebene Theorie (und sei es eine Alltagstheorie) interpretiert, haben sie der Feyerabend-Churchland'schen Vorstellung gemäß keine Bedeutung. Die Sätze können dann aber keine semantische Rolle spielen, indem sie etwa den Besitz von (empirischen) Begriffen ermöglichen würden, die mit erhöhter Wahrscheinlichkeit eine korrekte Beschreibung untersuchter Phänomene ermöglichen. Und selbst eine heuristische Rolle, die darin bestehen könnte, dass Beobachtungen von sich aus neuartige empirische Regularitäten nahe legen, scheint ausgeschlossen. Denn auch die heuristische Rolle für noch nicht in Betracht gezogene Theorien könnten sie nur spielen, wenn sie von sich aus eine Bedeutung der Art hätten, die Hinweise auf noch unentdeckte Theorien geben würde.

Daher können Feyerabend-Churchland-Beobachtungen nur empirisch zur Forschung beitragen, wenn schon eine interpretierende Theorie vorgegeben ist. Wenn es aber darum geht, zuallererst vielversprechende Hypothesen und Theorien zu formulieren, können solche Beobachtungen keine Beiträge leisten. Es erscheint dann aber unwahrscheinlich, dass wir angesichts der Unmenge denkbarer Theorien über einen Gegenstandbereich jemals dazu kommen sollen, eine überwiegend wahre Theorie auch nur zu formulieren. ${ }^{64}$

\section{c) Empirische Prüfung durch die Syntax von Beobachtungssätzen}

Damit stützt die syntaktische Theorie der Beobachtung und die implizierte Theorieabhängigkeit der Beobachtungsinhalte den epistemischen Pessimis-

${ }^{64}$ Man könnte versuchen, unabhängig von Erfahrung gegebene Einschränkungen für die in Betracht gezogenen Theorien anzunehmen, etwa natürliche Neigungen zu bestimmter Begriffsbildung, angeborene Begriffe oder Erkenntnisse a priori. Aber diese Einschränkungen müssten angesichts der großen Zahl möglicher Theorien massiv sein. Zudem ist unklar, ob wir so zu Theorien kommen, die eine höhere Wahrscheinlichkeit auf überwiegende Wahrheit haben. Weitgehende Annahmen in diese Richtung scheinen jedenfalls empiristisch nicht akzeptabel. 
mus auf die erste der beiden möglichen Weisen, die in Kapitel 1 unterschieden wurden. ${ }^{65}$ Schon die Formulierung einer überwiegend wahren Theorie wird durch diese Form der Theoriebeladenheit unwahrscheinlich gemacht. Aber auch auf die zweite Weise führt die syntaktische Theorie der Beobachtung zum Pessimismus. Feyerabend-Churchland-Beobachtungen machen die Möglichkeit pessimistischer Unterbestimmtheits-Szenarien wahrscheinlich. Wenn es eine systematisch tugendhafte Theorie gibt, die durch die Menge aller in einem Bereich aufstellbaren Beobachtungssätze empirisch bestätigbar ist, dann gibt es wahrscheinlich damit weitgehend unvereinbare Theorien, die mit derselben Menge von Beobachtungen vereinbar ist.

Denn Feyerabend-Churchland-Beobachtungen sind unabhängig von Theorien nur in ihrer Syntax (und dem Zeitpunkt ihrer Produktion) festgelegt. Selbst wenn man davon ausgeht, dass die Dispositionen zur Produktion von Beobachtungssätzen unabhängig von den geprüften Theorien sind, gibt es daher nur eine syntaktisch und durch den Zeitpunkt der Produktion bestimmte Menge von Beobachtungssätzen, die für alle alternativen Theorien gemeinsam ist. Die jeweiligen Theorien bestimmen dagegen vollständig die Interpretation der Beobachtungssätze. Daher können die Mengen der Beobachtungssätze ihrem Inhalt nach vor verschiedenen theoretischen Hintergründen weit divergieren.

Zwar wird nicht jede beliebige Theorie durch die Menge unabhängig produzierter und von ihr interpretierter Beobachtungssätze bestätigt werden. Wie Feyerabend zu Recht bemerkt, kann in einer Beobachtung beispielsweise auch die Negation eines Satzes, der aus der Theorie ableitbar ist, produziert werden. ${ }^{66}$ Es ist daher nicht garantiert, dass eine syntaktisch geprüfte Theorie empirisch bestätigt wird. Dies zeigt aber nur, dass für die Theorie ein Fehlschlagsrisiko besteht. Die Möglichkeit pessimistischer UnterbestimmtheitsSzenarien bleibt wahrscheinlich.

Denn für den Optimismus muss man annehmen, dass von den vielen denkbaren Theorien mindestens eine sowohl systematisch tugendhaft sein als auch durch die von ihr interpretierten Beobachtungssätze empirisch gestützt erscheinen kann. Da es demnach keine grundsätzlichen Hindernisse für die Erklärung einer solchen Beobachtungsmenge durch eine systematisch tugendhafte Theorie gibt, ist zu erwarten, dass auch einige alternative, weitgehend unvereinbare Theorien die Menge der Beobachtungssätze integrieren können. Hierfür ist lediglich erforderlich, dass die Beobachtungssätze, rein

65 Siehe Kap. 1, Abschn. 3c.

66 Siehe Feyerabend (1965), 213-215 für eine Darstellung des Zusammenspiels von Produktion und Interpretation Feyerabend'scher Beobachtungssätze und ihrer Rolle in der empirischen Prüfung von Theorien. 
syntaktisch spezifiziert, aus der Theorie ableitbar sind. Die Netzwerk-Semantik garantiert dann, dass die Sätze so interpretiert werden, dass sie auch inhaltlich die Theorie zu bestätigen scheinen. Es ist dann weder von einer empirisch gestützt erscheinenden Theorie noch von den von ihr interpretierten Beobachtungssätzen wahrscheinlich, dass sie überwiegend wahr sind. Auch wegen der wahrscheinlichen Möglichkeit solcher UnterbestimmtheitsSzenarien stützt die Theoriebeladenheit von Feyerabend-Churchland-Beobachtungen einen Pessimismus.

\section{Zusammenfassung}

Gegenstand dieses Kapitels ist eine Position zur Beschaffenheit und Theoriebeladenheit von Beobachtungen, die vor allem von Feyerabend und Churchland vertreten wird. Demnach sind erstens Beobachtungen im Allgemeinen als Kausalprozesse auffassbar, die zu Sätzen als wissenschaftlich relevanten Ergebnissen führen. Dabei soll sich die Kausalrelation nur an der Syntax der Sätze festmachen, so dass die Frage nach deren Semantik zunächst offen bleibt. Dieses nach dem Ablauf von Messprozessen gebildete Modell erwies sich insofern als problematisch, als es auch für Wahrnehmungen gelten soll. Denn Zustände perzeptuellen Erscheinens und die eingeschlossenen perzeptuellen Klassifikationen setzen für ihre Verursachung Fähigkeiten perzeptueller Klassifikation voraus, die mit dem vorgeschlagenen Modell nicht eingefangen werden können (Abschn. 2).

Die zweite zentrale Annahme der Position ist die Netzwerk-Semantik, derzufolge die Bedeutung der Beobachtungsausdrücke durch ein einbettendes Netz theoretischer Annahmen festgelegt wird (Abschn. 2a). Zwei Argumente hierfür wurden diskutiert. Das Argument von der Semantik theoretischer Ausdrücke setzt darauf, dass Beobachtungsausdrücke semantisch wie theoretische Ausdrücke funktionieren und dass für theoretische Ausdrücke eine Netzwerk-Semantik plausibel ist (Abschn. 3b). Als entscheidende Schwäche erwies sich die behauptete semantische Ähnlichkeit von theoretischen Termen und Beobachtungsausdrücken. So lässt sich die Sprache der fiktiven Gemeinschaft der Wärmestoff-Freunde gerade dann gut verstehen, wenn die Bedeutung ihrer Beobachtungsausdrücke an ihre perzeptuellen Fähigkeiten und nicht an ihre Theorien angebunden ist.

Das Ausschluss-Argument weist zunächst zwei alternative semantische Theorien für Beobachtungsausdrücke zurück, die Empfindungen bzw. nomologische Relationen zu Tatsachen oder Eigenschaften als semantisch konstitutiv auffassen. Es schließt dann daraus auf die Netzwerk-Semantik als verbleibende Option (Abschn. 3c). Die durchgeführte Diskussion ergab, dass die Zurückweisung der Alternativen plausibel ist. So könnte eine an Empfin- 
dungen anknüpfende Semantik von vornherein nur die Bedeutung von Ausdrücken für sinnliche Eigenschaften erklären. Eine auf nomologische Verknüpfungen setzende Semantik gerät dagegen durch die Möglichkeit umfassenden Irrtums in der Anwendung von Prädikaten in Schwierigkeiten. Allerdings ist das Ausschluss-Argument anfällig gegenüber der Formulierung einer weiteren Alternative, auf die ich aber erst in Kapitel 7 im Detail zu sprechen komme.

Die Auffassung von Beobachtungen und ihrer Theoriebeladenheit, die sich insgesamt ergibt, erwies sich für den epistemischen Optimismus als doppelt katastrophal. Erstens lässt sich mit nahe liegenden Zusatzannahmen dafür argumentieren, dass wir vermutlich niemals eine überwiegend wahre Theorie zu einem Bereich überhaupt empirisch zur Prüfung stellen werden (Abschn. 4b). Zweitens könnten wahrscheinlich viele weitgehend unvereinbare Theorien empirisch als gut gestützt erscheinen (Abschn. 4c). Beide Weisen der Stützung eines Pessimismus hängen daran, dass der diskutierten Position zufolge Beobachtungen nur hinsichtlich der Syntax von Beobachtungssätzen empirisch, in ihrer Semantik aber vollständig theoretisch festgelegt sind. Für eine Begründung des Optimismus muss daher gezeigt werden, dass diese extreme Form der Theoriebeladenheit unhaltbar ist. 